\title{
GROUND STATES FOR FRACTIONAL MAGNETIC OPERATORS
}

\author{
PIETRO D'AVENIA AND MARCO SQUASSINA
}

\begin{abstract}
We study a class of minimization problems for a nonlocal operator involving an external magnetic potential. The notions are physically justified and consistent with the case of absence of magnetic fields. Existence of solutions is obtained via concentration compactness.
\end{abstract}

\section{CONTEnTs}

1. Introduction and results 1

2. Functional setting 4

3. Preliminary stuff 8

4. Existence of minimizers 15

4.1. Subcritical symmetric case 15

4.2. Subcritical case 16

4.3. Critical case 19

References $\quad 21$

\section{INTRODUCTION AND RESULTS}

Since the late nineties, nonlocal integral operators like

$$
(-\Delta)^{s} u(x)=c_{s} \lim _{\varepsilon \searrow 0} \int_{B_{\varepsilon}^{c}(x)} \frac{u(x)-u(y)}{|x-y|^{3+2 s}} d y=\mathcal{F}^{-1}\left(|\xi|^{2 s} \mathcal{F}(u)(\xi)\right)(x), \quad u \in C_{c}^{\infty}\left(\mathbb{R}^{3}\right),
$$

where $s \in(0,1)$ and

$$
c_{s}=s 2^{2 s} \frac{\Gamma\left(\frac{3+2 s}{2}\right)}{\pi^{3 / 2} \Gamma(1-s)}
$$

being $\Gamma$ the Gamma function, have been widely used in the theory of Lévy processes. Indeed, in view of the Lévy-Khintchine formula, the generator $\mathscr{H}$ of the semigroup on $C_{c}^{\infty}\left(\mathbb{R}^{3}\right)$ associated to a general Lévy process is given by

$$
\mathscr{H} u(x)=-a_{i j} \partial_{x_{i} x_{j}}^{2} u(x)-b_{i} \partial_{x_{i}} u(x)-\lim _{\varepsilon \searrow 0} \int_{B_{\varepsilon}^{c}(0)}\left(u(x+y)-u(x)-1_{\{|y|<1\}}(y) y \cdot \nabla u(x)\right) d \mu
$$

with summation on repeated indexes and where $\mu$ is a Lévy nonnegative measure, namely

$$
\int_{\mathbb{R}^{3}} \frac{|y|^{2}}{1+|y|^{2}} d \mu<\infty .
$$

The last contribution in (1.2) represents the purely jump part of the Lévy process, while the first two terms represent a Brownian motion with drift. It is now well established that Lévy processes with jumps are more appropriate for some mathematical models in finance. Among Lévy processes, the only stochastically stable ones having jump part are those corresponding to radial measures as

$$
d \mu=\frac{c_{s}}{|y|^{3+2 s}} d y
$$

2010 Mathematics Subject Classification. 49A50, 26A33, 74G65, 82D99.

Key words and phrases. Fractional magnetic operators, minimization problems, concentration compactness. 
hence the importance of the definition (1.1). Moreover, the fractional Laplacian (1.1) allows to develop a generalization of quantum mechanics and also to describe the motion of a chain or array of particles that are connected by elastic springs and unusual diffusion processes in turbulent fluid motions and material transports in fractured media (for more details see e.g. $[1,9,22,26]$ and the references therein). Due to the results of Bourgain-Brézis-Mironescu [5,6], up to correcting the operator $(1.1)$ with the factor $(1-s)$ it follows that $(-\Delta)^{s} u$ converges to $-\Delta u$ in the limit $s \nearrow 1$. Thus, up to normalization, we may think the nonlocal case as an approximation of the local case. A pseudorelativistic extension of the Laplacian is the well known pseudodifferential operator $\sqrt{-\Delta+m^{2}}-m$ where $m$ is a nonnegative number. This operator appears in the study of free relativistic particles of mass $m$ and $\sqrt{-\Delta+m^{2}}$ is defined by $\mathcal{F}^{-1}\left(\sqrt{|\xi|^{2}+m^{2}} \mathcal{F}(u)(\xi)\right.$ ) (see [23] for more details). We observe that for $m=0$ we have the operator in (1.1) with $s=1 / 2$.

An important role in the study of particles which interact, e.g. using the Weyl covariant derivative, with a magnetic field $B=\nabla \times A, A: \mathbb{R}^{3} \rightarrow \mathbb{R}^{3}$, is assumed by another extension of the Laplacian, namely the magnetic Laplacian $(\nabla-\mathrm{i} A)^{2}$ (see $\left.[3,27]\right)$. Nonlinear magnetic Schrödinger equations like

$$
-(\nabla-\mathrm{i} A)^{2} u+u=f(u)
$$

have been extensively studied (see e.g. [2,8,12,15,21,28]).

In [19], Ichinose and Tamura, through oscillatory integrals, introduce the so-called Weyl pseudodifferential operator defined with mid-point prescription

$$
\begin{aligned}
\mathscr{H}_{A} u(x) & =\frac{1}{(2 \pi)^{3}} \int_{\mathbb{R}^{6}} e^{\mathrm{i}(x-y) \cdot \xi} \sqrt{\left|\xi-A\left(\frac{x+y}{2}\right)\right|^{2}+m^{2}} u(y) d y d \xi \\
& =\frac{1}{(2 \pi)^{3}} \int_{\mathbb{R}^{6}} e^{\mathrm{i}(x-y) \cdot\left(\xi+A\left(\frac{x+y}{2}\right)\right)} \sqrt{|\xi|^{2}+m^{2}} u(y) d y d \xi
\end{aligned}
$$

as a fractional relativistic generalization of the magnetic Laplacian (see also [17], the review article [18] and the references therein). The operator $\mathscr{H}_{A}$ takes the place of $\sqrt{-\Delta+m^{2}}$ and it is possible to show that for all $u \in C_{c}^{\infty}\left(\mathbb{R}^{3}, \mathbb{C}\right)$,

$$
\begin{aligned}
\mathscr{H}_{A} u(x) & =m u(x)-\lim _{\varepsilon \searrow 0} \int_{B_{\varepsilon}^{c}(0)}\left[e^{-\mathrm{i} y \cdot A\left(x+\frac{y}{2}\right)} u(x+y)-u(x)-1_{\{|y|<1\}}(y) y \cdot(\nabla-\mathrm{i} A(x)) u(x)\right] d \mu \\
& =m u(x)+\lim _{\varepsilon \searrow 0} \int_{B_{\varepsilon}^{c}(x)}\left[u(x)-e^{\mathrm{i}(x-y) \cdot A\left(\frac{x+y}{2}\right)} u(y)\right] \mu(y-x) d y
\end{aligned}
$$

where

$$
d \mu=\mu(y) d y= \begin{cases}2\left(\frac{m}{2 \pi}\right)^{2} \frac{K_{2}(m|y|)}{|y|^{2}} d y, & m>0, \\ \frac{1}{\pi^{2}|y|^{4}} d y, & m=0,\end{cases}
$$

and $K_{2}$ is the modified Bessel function of the third kind of order 2 (see e.g. [18, Subsection 3.1]). In this paper we are concerned with the operator

$$
(-\Delta)_{A}^{s} u(x)=c_{s} \lim _{\varepsilon \searrow 0} \int_{B_{\varepsilon}^{c}(x)} \frac{u(x)-e^{\mathrm{i}(x-y) \cdot A\left(\frac{x+y}{2}\right)} u(y)}{|x-y|^{3+2 s}} d y, \quad x \in \mathbb{R}^{3},
$$

and, in particular, with ground state solutions of the equation

$$
\left(\mathscr{P}_{s, A}\right) \quad(-\Delta)_{A}^{s} u+u=|u|^{p-2} u \text { in } \mathbb{R}^{3} \text {. }
$$

The operator (1.3) is consistent with the definition of fractional Laplacian given in (1.1) if $A=0$ and with $\mathscr{H}_{A}$ for $m=0$ and $s=1 / 2$. To our knowledge, this is the first mathematical contribution to the study of nonlinear problems involving operator (1.3).

For the sake of completeness we mention that there exist other different definitions of the magnetic pseudorelativistic operator (see [18,20,23]) and in [16] a fractional magnetic operator 
$(\nabla-\mathrm{i} A)^{2 s}$ is defined through the spectral theorem (see also discussion on the different definitions in $[18$, Proposition 2.6]).

Throughout the paper we consider magnetic potentials $A$ 's which have locally bounded gradient. We now state our results.

Let $2<p<6 /(3-2 s)$ and consider the minimization problem

$$
\mathscr{M}_{A}=\inf _{u \in \mathscr{S}}\left(\int_{\mathbb{R}^{3}}|u|^{2} d x+\frac{c_{s}}{2} \int_{\mathbb{R}^{6}} \frac{\left|e^{-\mathrm{i}(x-y) \cdot A\left(\frac{x+y}{2}\right)} u(x)-u(y)\right|^{2}}{|x-y|^{3+2 s}} d x d y\right),
$$

where

$$
\mathscr{S}=\left\{u \in H_{A}^{s}\left(\mathbb{R}^{3}, \mathbb{C}\right): \int_{\mathbb{R}^{3}}|u|^{p} d x=1\right\}
$$

and $H_{A}^{s}\left(\mathbb{R}^{3}, \mathbb{C}\right)$ is a suitable Hilbert space defined in Section 2. Once a solution to $\mathscr{M}_{A}$ exists, due to the Lagrange Multiplier Theorem, we get a weak solution to $\left(\mathscr{P}_{s, A}\right)$, see Sections 2 and 4. When $\mathscr{S}$ is restricted to radially symmetric functions, the problem is denoted by $\mathscr{M}_{A, r}$.

First we give the following

Definition 1.1. We say that $A$ satisfies assumption $\mathscr{A}$, if for any unbounded sequence $\Xi=$ $\left\{\xi_{n}\right\}_{n \in \mathbb{N}} \subset \mathbb{R}^{3}$ there exist a sequence $\left\{H_{n}\right\}_{n \in \mathbb{N}} \subset \mathbb{R}^{3}$ and a function $A_{\Xi}: \mathbb{R}^{3} \rightarrow \mathbb{R}^{3}$ such that

$$
\lim _{n} A_{n}(x)=A_{\Xi}(x) \text { for all } x \in \mathbb{R}^{3} \text { and } \sup _{n}\left\|A_{n}\right\|_{L^{\infty}(K)}<\infty \text { for all compact sets } K,
$$

where $A_{n}(x):=A\left(x+\xi_{n}\right)+H_{n}$ and $\left\{\xi_{n}\right\}$ is a subsequence of $\Xi$ such that $\left|\xi_{n}\right| \rightarrow \infty$.

We also set $\mathscr{X}:=\left\{\Xi=\left\{\xi_{n}\right\}_{n \in \mathbb{N}}\right.$ unbounded : condition (1.4) holds $\}$. Observe that, if $A$ admits limit as $|x| \rightarrow \infty$, then it satisfies assumption $\mathscr{A}$.

Our main result is

Theorem 1.2 (Subcritical case). The following facts hold:

(i) $\mathscr{M}_{A, r}$ has a solution;

(ii) if $A$ is linear, then $\mathscr{M}_{A}$ has a solution;

(iii) if $A$ satisfies $\mathscr{A}$ and $\mathscr{M}_{A}<\inf _{\Xi \in \mathscr{X}} \mathscr{M}_{A_{\Xi}}$, then $\mathscr{M}_{A}$ has a solution.

We also consider the minimization problem

$$
\mathscr{M}_{A}^{c}:=\inf _{u \in \mathscr{S}^{c}} \frac{c_{s}}{2} \int_{\mathbb{R}^{6}} \frac{\left|e^{-\mathrm{i}(x-y) \cdot A\left(\frac{x+y}{2}\right)} u(x)-u(y)\right|^{2}}{|x-y|^{3+2 s}} d x d y,
$$

where

$$
\mathscr{S}^{c}=\left\{u \in D_{A}^{s}\left(\mathbb{R}^{3}, \mathbb{C}\right): \int_{\mathbb{R}^{3}}|u|^{6 /(3-2 s)} d x=1\right\}
$$

and $D_{A}^{s}\left(\mathbb{R}^{3}, \mathbb{C}\right)$ is a suitable Hilbert space defined in Subection 4.3. We are able to prove

Theorem 1.3 (Critical case). The following facts hold:

(i) if $\mathscr{M}_{A}^{c}$ has a solution $u$, there exist $z \in \mathbb{R}^{3}, \varepsilon>0$ and $\vartheta_{A}: \mathbb{R}^{3} \rightarrow \mathbb{R}$ such that

$$
u(x)=d_{s}\left(\frac{\varepsilon}{\varepsilon^{2}+|x-z|^{2}}\right)^{\frac{3-2 s}{2}} e^{\mathrm{i} \vartheta_{A}(x)} ;
$$

(ii) if for some $k \in \mathbb{N}$ and $E \subset \mathbb{R}^{6}$ of positive measure

$$
(x-y) \cdot A\left(\frac{x+y}{2}\right) \not \equiv 2 k \pi \quad \text { for all }(x, y) \in E,
$$

then $\mathscr{M}_{A}^{c}$ has no solution $u$ of the form $e^{\mathrm{i} \vartheta} v(x)$ where $\vartheta \in \mathbb{R}$ and $v$ of fixed sign. 
The local version of the above results can be found in the work [15] by Esteban and Lions. In [14], for the case without magnetic field and with subcritical nonlinearities, existence of ground states was obtained using different arguments, namely without involving concentration compactness arguments, but instead symmetrizing the minimizing sequences, by using

$$
\int_{\mathbb{R}^{6}} \frac{\left.|| u(x)\right|^{*}-\left.|u(y)|^{*}\right|^{2}}{|x-y|^{3+2 s}} d x d y \leq \int_{\mathbb{R}^{6}} \frac{|u(x)-u(y)|^{2}}{|x-y|^{3+2 s}} d x d y,
$$

for all $u \in H^{s}\left(\mathbb{R}^{3}\right)$, where $v^{*}$ denotes the Schwarz symmetrization of $v: \mathbb{R}^{3} \rightarrow \mathbb{R}^{+}$. On the contrary, when $A \not \equiv 0$, the inequality

$$
\int_{\mathbb{R}^{6}} \frac{\left.\left|e^{-\mathrm{i}(x-y) \cdot A\left(\frac{x+y}{2}\right)}\right| u(x)\right|^{*}-\left.|u(y)|^{*}\right|^{2}}{|x-y|^{3+2 s}} d x d y \leq \int_{\mathbb{R}^{6}} \frac{\left|e^{-\mathrm{i}(x-y) \cdot A\left(\frac{x+y}{2}\right)} u(x)-u(y)\right|^{2}}{|x-y|^{3+2 s}} d x d y,
$$

does not seem to work and a different strategy for the proof has to be outlined. Dealing with the nonlocal case, it is natural to expect that, in the study of minimizing sequences, the hardest stage is that of ruling out the dichotomy in the concentration compactness alternative. This is in fact the case, but thanks to a careful analysis developed in Lemma 3.9, dichotomy can be ruled out allowing for tightness and hence the strong convergence of minimizing sequences up translations and phase changes.

We organize the paper in the following way: in Section 2 we introduce the functional setting of the problem and we provide some basic properties about it; in Section 3 we show further technical facts on the functional setting as well as some preliminary results about the ConcentrationCompactness procedure; finally, in Section 4, we complete with the proofs of our results.

Acknowledgments. The research was partially supported by Gruppo Nazionale per l'Analisi Matematica, la Probabilità e le loro Applicazioni (INdAM).

Notations. We denote by $B_{R}(\xi)$ a ball in $\mathbb{R}^{3}$ of center $\xi$ and radius $R$. For a measurable set $E \subset \mathbb{R}^{3}$ we denote by $E^{c}$ the complement of $E$ in $\mathbb{R}^{3}$, namely $E^{c}=\mathbb{R}^{3} \backslash E$. We denote by $1_{E}$ the indicator function of $E$. The symbol $\mathcal{L}^{n}(\Omega)$ stands for the Lebesgue measure of a measurable subset $\Omega \subset \mathbb{R}^{n}$. For a complex number $z \in \mathbb{C}$, the symbol $\Re z$ indicates its real part and $\Im z$ its imaginary part. The modulus of $z$ is denoted by $|z|$. The standard norm of $L^{p}$ spaces is denoted by $\|\cdot\|_{L^{p}}$.

\section{FunCtional Setting}

Let $L^{2}\left(\mathbb{R}^{3}, \mathbb{C}\right)$ be the Lebesgue space of complex valued functions with summable square endowed with the real scalar product

$$
\langle u, v\rangle_{L^{2}}:=\Re \int_{\mathbb{R}^{3}} u \bar{v} d x, \quad \text { for all } u, v \in L^{2}\left(\mathbb{R}^{3}, \mathbb{C}\right),
$$

and $A: \mathbb{R}^{3} \rightarrow \mathbb{R}^{3}$ be a continuous function. We consider the magnetic Gagliardo semi-norm defined by

$$
[u]_{s, A}^{2}:=\frac{c_{s}}{2} \int_{\mathbb{R}^{6}} \frac{\left|e^{-\mathrm{i}(x-y) \cdot A\left(\frac{x+y}{2}\right)} u(x)-u(y)\right|^{2}}{|x-y|^{3+2 s}} d x d y,
$$

the scalar product defined by

$$
\langle u, v\rangle_{s, A}:=\langle u, v\rangle_{L^{2}}+\frac{c_{s}}{2} \Re \int_{\mathbb{R}^{6}} \frac{\left(e^{-\mathrm{i}(x-y) \cdot A\left(\frac{x+y}{2}\right)} u(x)-u(y)\right) \overline{\left(e^{-\mathrm{i}(x-y) \cdot A\left(\frac{x+y}{2}\right)} v(x)-v(y)\right)}}{|x-y|^{3+2 s}} d x d y,
$$


and the corresponding norm denoted by

$$
\|u\|_{s, A}:=\left(\|u\|_{L^{2}}^{2}+[u]_{s, A}^{2}\right)^{1 / 2} .
$$

We consider the space $\mathcal{H}$ of measurable functions $u: \mathbb{R}^{3} \rightarrow \mathbb{C}$ such that $\|u\|_{s, A}<\infty$.

Proposition 2.1. $\left(\mathcal{H},\langle\cdot, \cdot\rangle_{s, A}\right)$ is a real Hilbert space.

Proof. It is readily checked that $\langle u, v\rangle_{s, A}$ is a real scalar product. Let us prove that $\mathcal{H}$ with this scalar product is complete. Let $\left\{u_{n}\right\}_{n \in \mathbb{N}}$ be a Cauchy sequence in $\mathcal{H}$, namely for every $\varepsilon>0$ there exists $\nu_{\varepsilon} \in \mathbb{N}$ such that for all $m, n>\nu_{\varepsilon}$ we have $\left\|u_{n}-u_{m}\right\|_{s, A}<\varepsilon$. Thus $\left\{u_{n}\right\}_{n \in \mathbb{N}}$ is a Cauchy sequence on $L^{2}\left(\mathbb{R}^{3}, \mathbb{C}\right)$ and then there exists $u \in L^{2}\left(\mathbb{R}^{3}, \mathbb{C}\right)$ such that $u_{n} \rightarrow u$ in $L^{2}\left(\mathbb{R}^{3}, \mathbb{C}\right)$ and a.e. in $\mathbb{R}^{3}$. Firstly, we prove that $u \in \mathcal{H}$. By Fatou Lemma we have

$$
[u]_{s, A}^{2} \leq \liminf _{n}\left[u_{n}\right]_{s, A}^{2} \leq \liminf _{n}\left(\left[u_{n}-u_{\nu_{1}}\right]_{s, A}+\left[u_{\nu_{1}}\right]_{s, A}\right)^{2} \leq\left(1+\left[u_{\nu_{1}}\right]_{s, A}\right)^{2} .
$$

Thus it remains to prove that $\left[u_{n}-u\right]_{s, A} \rightarrow 0$ as $n \rightarrow \infty$. Again, by Fatou Lemma

$$
\left[u_{n}-u\right]_{s, A} \leq \liminf _{k}\left[u_{n}-u_{k}\right]_{s, A} \leq \liminf _{k}\left\|u_{n}-u_{k}\right\|_{s, A} \leq \varepsilon
$$

for all $\varepsilon>0$ and $n$ large.

For any function $w: \mathbb{R}^{3} \rightarrow \mathbb{C}$ and a.e. $x \in \mathbb{R}^{3}$, we set

$$
w_{x}(y):=e^{\mathrm{i}(x-y) \cdot A\left(\frac{x+y}{2}\right)} w(y), \quad \text { for } y \in \mathbb{R}^{3} .
$$

We have

Proposition 2.2. The space $C_{c}^{\infty}\left(\mathbb{R}^{3}, \mathbb{C}\right)$ is a subspace of $\mathcal{H}$.

Proof. It is enough to prove that $[u]_{s, A}<\infty$, for any $u \in C_{c}^{\infty}\left(\mathbb{R}^{3}, \mathbb{C}\right)$. If $K$ is the compact support of $u$, we have

$$
\int_{\mathbb{R}^{6}} \frac{\left|e^{-\mathrm{i}(x-y) \cdot A\left(\frac{x+y}{2}\right)} u(x)-u(y)\right|^{2}}{|x-y|^{3+2 s}} d x d y \leq 2 \int_{K \times \mathbb{R}^{3}} \frac{\left|u_{x}(x)-u_{x}(y)\right|^{2}}{|x-y|^{3+2 s}} d x d y .
$$

Observe that, since $\nabla A$ is locally bounded, the gradient of the function $(x, y) \mapsto u_{x}(y)$ is bounded on $K \times \mathbb{R}^{3}$. Then we have $\left|u_{x}(x)-u_{x}(y)\right| \leq C|x-y|$ for any $(x, y) \in K \times \mathbb{R}^{3}$. Of course, we also have $\left|u_{x}(x)-u_{x}(y)\right| \leq C$ for any $(x, y) \in K \times \mathbb{R}^{3}$. Hence, we get

$$
\begin{aligned}
\int_{K \times \mathbb{R}^{3}} \frac{\left|u_{x}(x)-u_{x}(y)\right|^{2}}{|x-y|^{3+2 s}} d x d y & \leq C \int_{K \times \mathbb{R}^{3}} \frac{\min \left\{|x-y|^{2}, 1\right\}}{|x-y|^{3+2 s}} d x d y \\
& \leq C \int_{B_{1}(0)} \frac{1}{|z|^{1+2 s}} d z+C \int_{B_{1}^{c}(0)} \frac{1}{|z|^{3+2 s}} d z
\end{aligned}
$$

which concludes the proof.

Thus we can give the following

Definition 2.3. We define $H_{A}^{s}\left(\mathbb{R}^{3}, \mathbb{C}\right)$ as the closure of $C_{c}^{\infty}\left(\mathbb{R}^{3}, \mathbb{C}\right)$ in $\mathcal{H}$.

Then, $H_{A}^{s}\left(\mathbb{R}^{3}, \mathbb{C}\right)$ is a real Hilbert space by Proposition 2.1. For $A=0$ this space is consistent with the usual fractional space $H^{s}\left(\mathbb{R}^{3}, \mathbb{C}\right)$ whose norm is denoted by $\|\cdot\|_{s}$. For a given Lebesgue measurable set $E \subset \mathbb{R}^{3}$ the localized Gagliardo norms are defined by

$$
\begin{aligned}
\|u\|_{H_{A}^{s}(E)} & :=\left(\int_{E}|u(x)|^{2} d x+\frac{c_{s}}{2} \int_{E \times E} \frac{\left|e^{-\mathrm{i}(x-y) \cdot A\left(\frac{x+y}{2}\right)} u(x)-u(y)\right|^{2}}{|x-y|^{3+2 s}} d x d y\right)^{1 / 2}, \\
\|u\|_{H^{s}(E)} & :=\left(\int_{E}|u(x)|^{2} d x+\frac{c_{s}}{2} \int_{E \times E} \frac{|u(x)-u(y)|^{2}}{|x-y|^{3+2 s}} d x d y\right)^{1 / 2} .
\end{aligned}
$$


The operator $(-\Delta)_{A}^{s}: H_{A}^{s}\left(\mathbb{R}^{3}, \mathbb{C}\right) \rightarrow H_{A}^{-s}\left(\mathbb{R}^{3}, \mathbb{C}\right)$ is defined by duality as

$$
\begin{aligned}
\left\langle(-\Delta)_{A}^{s} u, v\right\rangle & :=\frac{c_{s}}{2} \Re \int_{\mathbb{R}^{6}} \frac{\left(e^{-\mathrm{i}(x-y) \cdot A\left(\frac{x+y}{2}\right)} u(x)-u(y)\right) \overline{\left(e^{-\mathrm{i}(x-y) \cdot A\left(\frac{x+y}{2}\right)} v(x)-v(y)\right)}}{|x-y|^{3+2 s}} d x d y \\
& =\frac{c_{s}}{2} \Re \int_{\mathbb{R}^{6}} \frac{\left(u(x)-e^{\mathrm{i}(x-y) \cdot A\left(\frac{x+y}{2}\right)} u(y)\right)}{|x-y|^{3+2 s}} d x d y .
\end{aligned}
$$

If $f \in H_{A}^{-s}\left(\mathbb{R}^{3}, \mathbb{C}\right)$, we say that $u \in H_{A}^{s}\left(\mathbb{R}^{3}, \mathbb{C}\right)$ is a weak solution to

$$
(-\Delta)_{A}^{s} u+u=f, \quad \text { in } \mathbb{R}^{3},
$$

if we have

$$
\begin{aligned}
& \frac{c_{s}}{2} \Re \int_{\mathbb{R}^{6}} \frac{\left(u(x)-e^{\mathrm{i}(x-y) \cdot A\left(\frac{x+y}{2}\right)} u(y)\right) \overline{\left(v(x)-e^{\mathrm{i}(x-y) \cdot A\left(\frac{x+y}{2}\right)} v(y)\right)}}{|x-y|^{3+2 s}} d x d y \\
& +\Re \int_{\mathbb{R}^{3}} u \bar{v} d x=\Re \int_{\mathbb{R}^{3}} f \bar{v} d x, \quad \text { for all } v \in H_{A}^{s}\left(\mathbb{R}^{3}, \mathbb{C}\right) .
\end{aligned}
$$

Of course, one can equivalently define the weak solution by testing over functions $v \in C_{c}^{\infty}\left(\mathbb{R}^{3}, \mathbb{C}\right)$. On smooth functions, the operator $(-\Delta)_{A}^{s}$ admits the point-wise representation (1.3). To show this we need the following preliminary results.

Lemma 2.4. Let $K$ be a compact subset of $\mathbb{R}^{3}, R>0$ and set $K^{\prime}=\left\{x \in \mathbb{R}^{3}: d(x, K) \leq R\right\}$. Assume that $f \in C^{2}\left(\mathbb{R}^{6}\right)$ and that $g \in C^{1, \gamma}\left(K^{\prime}\right)$ for some $\gamma \in[0,1]$. If $h(x, y)=f(x, y) g(y)$, then there exists a positive constant $C$ depending on $K, f, g, R$, such that

$$
\left|\nabla_{y} h\left(x, y_{2}\right)-\nabla_{y} h\left(x, y_{1}\right)\right| \leq C\left|y_{2}-y_{1}\right|^{\gamma},
$$

for all $x \in K$ and every $y_{2}, y_{1} \in K^{\prime}$.

Proof. The proof is omitted as it is straightforward.

Lemma 2.5. Let $A \in C^{2}\left(\mathbb{R}^{3}\right)$ and $u \in C_{\text {loc }}^{1, \gamma}\left(\mathbb{R}^{3}, \mathbb{C}\right)$ for some $\gamma \in[0,1]$. Then, for any compact set $K \subset \mathbb{R}^{3}$ and $R>0$, there exists a positive constant $C$ depending on $R, K, A, u$, such that

$$
\left|u_{x}(x+y)+u_{x}(x-y)-2 u_{x}(x)\right| \leq C|y|^{1+\gamma},
$$

for every $x \in K$ and $y \in B_{R}(0)$.

Proof. Fix a compact set $K \subset \mathbb{R}^{3}$ and $R>0$. Consider $x \in K$ and $y \in B_{R}(0)$. Then, by the Mean Value Theorem, there exist $\tau_{1}, \tau_{2} \in[0,1]$ such that

$$
\begin{aligned}
\left|u_{x}(x+y)+u_{x}(x-y)-2 u_{x}(x)\right| & =\left|\nabla_{y} u_{x}\left(x+\tau_{1} y\right) \cdot y-\nabla_{y} u_{x}\left(x-\tau_{2} y\right) \cdot y\right| \\
& \leq\left|\nabla_{y} u_{x}\left(x+\tau_{1} y\right)-\nabla_{y} u_{x}\left(x-\tau_{2} y\right)\right||y| \leq C|y|^{1+\gamma}
\end{aligned}
$$

where in the last inequality we use Lemma 2.4 with $f(x, y)=e^{\mathrm{i}(x-y) \cdot A\left(\frac{x+y}{2}\right)}$ and $g(y)=u(y)$.

Thus in the case $u$ and $A$ are smooth enough, we have the following result

Theorem 2.6 (Weak to strong solution). Let $u \in H_{A}^{s}\left(\mathbb{R}^{3}, \mathbb{C}\right)$ be a weak solution to (2.2). Assume that $A \in C^{2}\left(\mathbb{R}^{3}\right)$ and that

$$
u \in L^{\infty}\left(\mathbb{R}^{3}, \mathbb{C}\right) \cap C_{\mathrm{loc}}^{1, \gamma}\left(\mathbb{R}^{3}, \mathbb{C}\right), \quad \text { for some } \gamma \in(0,1] \text { with } \gamma>2 s-1 .
$$

Then $u$ solves problem (2.2) pointwise a.e. in $\mathbb{R}^{3}$. 
Proof. With the notation introduced in (2.1), the definition of weak solution writes as

$$
\frac{c_{s}}{2} \Re \int_{\mathbb{R}^{6}} \frac{\left(u_{x}(x)-u_{x}(y)\right) \overline{\left(v_{x}(x)-v_{x}(y)\right)}}{|x-y|^{3+2 s}} d x d y+\Re \int_{\mathbb{R}^{3}} u \bar{v} d x=\Re \int_{\mathbb{R}^{3}} f \bar{v} d x,
$$

for all $v \in C_{c}^{\infty}\left(\mathbb{R}^{3}, \mathbb{C}\right)$. Let us fix a $v \in C_{c}^{\infty}\left(\mathbb{R}^{3}, \mathbb{C}\right)$ and set $K:=\operatorname{supp}(v)$. Now, for any $\varepsilon>0$, we introduce the auxiliary function $g_{\varepsilon}: K \rightarrow \mathbb{R}$ defined by

$$
g_{\varepsilon}(x):=\frac{c_{s}}{2} \int_{\mathbb{R}^{3}} \frac{u_{x}(x)-u_{x}(y)}{|x-y|^{3+2 s}} 1_{B_{\varepsilon}^{c}(x)}(y) d y .
$$

Note that for all $x \in K$ we have that

$$
g_{\varepsilon}(x) \rightarrow \frac{1}{2}(-\Delta)_{A}^{s} u(x), \quad \text { as } \varepsilon \rightarrow 0 \text { whenever the limit exists. }
$$

Simple changes of variables show that $g_{\varepsilon}$ can be equivalently written as

$$
g_{\varepsilon}(x)=-\frac{c_{s}}{4} \int_{\mathbb{R}^{3}} \frac{u_{x}(x+y)+u_{x}(x-y)-2 u_{x}(x)}{|y|^{3+2 s}} 1_{B_{\varepsilon}^{c}(0)}(y) d y .
$$

Furthermore, by Lemma 2.5, there exist $C>0$ and $R>0$ such that

$$
\left|u_{x}(x+y)+u_{x}(x-y)-2 u_{x}(x)\right| \leq C|y|^{1+\gamma}, \quad \text { for } x \in K \text { and } y \in B_{R}(0) .
$$

Therefore, taking into account that $\left|u_{x}(y)\right| \leq\|u\|_{L^{\infty}}$ for all $y \in \mathbb{R}^{3}$, we have the inequality

$$
\frac{\left|u_{x}(x+y)+u_{x}(x-y)-2 u_{x}(x)\right|}{|y|^{3+2 s}} \leq \frac{C}{|y|^{2+2 s-\gamma}} 1_{B_{R}(0)}(y)+\frac{C}{|y|^{3+2 s}} 1_{B_{R}^{c}(0)}(y),
$$

for some constant $C$. Due to the assumption $\gamma>2 s-1$, the right hand side belongs to $L^{1}\left(\mathbb{R}^{3}\right)$. Then, by dominated convergence, the limit of $g_{\varepsilon}(x)$ as $\varepsilon \rightarrow 0$ exists a.e. in $K$ and it is thus equal to $\frac{1}{2}(-\Delta)_{A}^{s} u(x)$ by $(2.4)$. Since also $\left|g_{\varepsilon}(x)\right| \leq C$ a.e. in $K$, again the dominated convergence yields

$$
g_{\varepsilon} \rightarrow \frac{1}{2}(-\Delta)_{A}^{s} u, \quad \text { strongly in } L^{1}(K) .
$$

Now, the first term in formula (2.3) can be treated as follows

$$
\begin{aligned}
& \frac{c_{s}}{2} \int_{\mathbb{R}^{6}} \frac{\left(u_{x}(x)-u_{x}(y)\right) \overline{\left(v_{x}(x)-v_{x}(y)\right)}}{|x-y|^{3+2 s}} d x d y \\
& =\lim _{\varepsilon \rightarrow 0} \frac{c_{s}}{2} \int_{\mathbb{R}^{6}} \frac{\left(u_{x}(x)-u_{x}(y)\right) \overline{\left(v_{x}(x)-v_{x}(y)\right)}}{|x-y|^{3+2 s}} 1_{B_{\varepsilon}^{c}(x)}(y) d x d y \\
& =\lim _{\varepsilon \rightarrow 0}\left(\int_{\mathbb{R}^{3}} g_{\varepsilon}(x) \overline{v(x)} d x-\frac{c_{s}}{2} \int_{\mathbb{R}^{6}} \frac{\left(u_{x}(x)-u_{x}(y)\right) \overline{v_{x}(y)}}{|x-y|^{3+2 s}} 1_{B_{\varepsilon}^{c}(x)}(y) d x d y\right) .
\end{aligned}
$$

By Fubini Theorem on the second term of the last equality, switching the two variables and observing that

$$
-\left(u_{y}(y)-u_{y}(x)\right) \overline{v_{y}(x)} 1_{B_{\varepsilon}^{c}(y)}(x)=\left(u_{x}(x)-u_{x}(y)\right) \overline{v(x)} 1_{B_{\varepsilon}^{c}(x)}(y)
$$

yields

$$
\frac{c_{s}}{2} \int_{\mathbb{R}^{6}} \frac{\left(u_{x}(x)-u_{x}(y)\right) \overline{\left(v_{x}(x)-v_{x}(y)\right)}}{|x-y|^{3+2 s}} d x d y=\lim _{\varepsilon \rightarrow 0} \int_{\mathbb{R}^{3}} 2 g_{\varepsilon}(x) \overline{v(x)} d x=\int_{\mathbb{R}^{3}}(-\Delta)_{A}^{s} u(x) \overline{v(x)} d x,
$$

where we used (2.5) in the last equality. Then, from formula (2.3), we conclude that

$$
\Re\left(\int_{\mathbb{R}^{3}}\left((-\Delta)_{A}^{s} u+u-f\right) \bar{v} d x\right)=0, \quad \text { for all } v \in C_{c}^{\infty}\left(\mathbb{R}^{3}, \mathbb{C}\right),
$$

yielding $(-\Delta)_{A}^{s} u+u=f$ a.e. in $\mathbb{R}^{3}$. The proof is complete. 
We conclude the section with an observation about the formal consistency of the spaces $H_{A}^{s}\left(\mathbb{R}^{3}, \mathbb{C}\right)$, up to suitably correcting the norm, with the usual local Sobolev spaces without magnetic field in the singular limit as $s \rightarrow 1$ and $A \rightarrow 0$ pointwise. Consider the modified norm

$$
\|u\|_{s, A}:=\left(\|u\|_{L^{2}}^{2}+(1-s)[u]_{s, A}^{2}\right)^{1 / 2}
$$

By arguing as in the proof of Lemma 4.6, it follows that

$$
\lim _{A \rightarrow 0}[u]_{s, A}^{2}=[u]_{s, 0}^{2}, \quad \text { for all } u \in C_{c}^{\infty}\left(\mathbb{R}^{3}, \mathbb{C}\right) .
$$

Moreover, from the results of Brezis-Bourgain-Mironescu [5,6], we know that

$$
\lim _{s \rightarrow 1}(1-s)[u]_{s, 0}^{2}=\|\nabla u\|_{L^{2}}^{2}, \quad \text { for all } u \in C_{c}^{\infty}\left(\mathbb{R}^{3}, \mathbb{C}\right) .
$$

In conclusion

$$
\lim _{s \rightarrow 1} \lim _{A \rightarrow 0} \mid\|u\|_{s, A}=\|u\|_{H^{1}\left(\mathbb{R}^{3}\right)}, \quad \text { for all } u \in C_{c}^{\infty}\left(\mathbb{R}^{3}, \mathbb{C}\right) .
$$

Hence $\||u|\|_{s, A}$ approximates the $H^{1}$-norm for $s \sim 1$ and $A \sim 0$.

\section{Preliminary StufF}

In this section we provide some technical facts about the functional setting of the problem as well as some preliminary results about the Concentration-Compactness procedure.

Lemma 3.1 (Diamagnetic inequality). For every $u \in H_{A}^{s}\left(\mathbb{R}^{3}, \mathbb{C}\right)$ it holds $|u| \in H^{s}\left(\mathbb{R}^{3}\right)$. More precisely

$$
\||u|\|_{s} \leq\|u\|_{s, A}, \quad \text { for every } u \in H_{A}^{s}\left(\mathbb{R}^{3}, \mathbb{C}\right) .
$$

Proof. For a.e. $x, y \in \mathbb{R}^{3}$ we have

$$
\Re\left(e^{-\mathrm{i}(x-y) \cdot A\left(\frac{x+y}{2}\right)} u(x) \overline{u(y)}\right) \leq|u(x)||u(y)| .
$$

Therefore, we have

$$
\begin{aligned}
\left|e^{-\mathrm{i}(x-y) \cdot A\left(\frac{x+y}{2}\right)} u(x)-u(y)\right|^{2} & =|u(x)|^{2}+|u(y)|^{2}-2 \Re\left(e^{-\mathrm{i}(x-y) \cdot A\left(\frac{x+y}{2}\right)} u(x) \overline{u(y)}\right) \\
& \geq|u(x)|^{2}+|u(y)|^{2}-2|u(x)||u(y)|=\left.|| u(x)|-| u(y)\right|^{2},
\end{aligned}
$$

which immediately yields the assertion.

Remark 3.2 (Pointwise Diamagnetic inequality). There holds

$$
|| u(x)|-| u(y)|| \leq\left|e^{-\mathrm{i}(x-y) \cdot A\left(\frac{x+y}{2}\right)} u(x)-u(y)\right|, \quad \text { for a.e. } x, y \in \mathbb{R}^{3} .
$$

We have the following local embedding of $H_{A}^{s}\left(\mathbb{R}^{3}, \mathbb{C}\right)$.

Lemma 3.3 (Local embedding in $H^{s}\left(\mathbb{R}^{3}, \mathbb{C}\right)$ ). For every compact set $K \subset \mathbb{R}^{3}$, the space $H_{A}^{s}\left(\mathbb{R}^{3}, \mathbb{C}\right)$ is continuously embedded into $H^{s}(K, \mathbb{C})$.

Proof. Fixed a compact $K \subset \mathbb{R}^{3}$, we have

$$
\begin{aligned}
\|u\|_{H^{s}(K)}^{2}= & \int_{K}|u(x)|^{2} d x+\frac{c_{s}}{2} \int_{K \times K} \frac{|u(x)-u(y)|^{2}}{|x-y|^{3+2 s}} d x d y \\
\leq & \int_{\mathbb{R}^{3}}|u(x)|^{2} d x+C \int_{K \times K} \frac{\left|e^{-\mathrm{i}(x-y) \cdot A\left(\frac{x+y}{2}\right)} u(x)-u(y)\right|^{2}}{|x-y|^{3+2 s}} d x d y \\
& +C \int_{K \times K} \frac{|u(x)|^{2}\left|e^{-\mathrm{i}(x-y) \cdot A\left(\frac{x+y}{2}\right)}-1\right|^{2}}{|x-y|^{3+2 s}} d x d y \\
\leq & C\|u\|_{s, A}^{2}+C J,
\end{aligned}
$$


where we have set

$$
J:=\int_{K \times K} \frac{|u(x)|^{2}\left|e^{-\mathrm{i}(x-y) \cdot A\left(\frac{x+y}{2}\right)}-1\right|^{2}}{|x-y|^{3+2 s}} d x d y .
$$

We now prove that $J \leq C\|u\|_{L^{2}}^{2}$, which ends the proof. We have

$$
\begin{aligned}
J= & \int_{K}|u(x)|^{2} \int_{K \cap\{|x-y| \geq 1\}} \frac{\left|e^{-\mathrm{i}(x-y) \cdot A\left(\frac{x+y}{2}\right)}-1\right|^{2}}{|x-y|^{3+2 s}} d x d y \\
& +\int_{K}|u(x)|^{2} \int_{K \cap\{|x-y| \leq 1\}} \frac{\left|e^{-\mathrm{i}(x-y) \cdot A\left(\frac{x+y}{2}\right)}-1\right|^{2}}{|x-y|^{3+2 s}} d x d y \\
\leq & C \int_{K}|u(x)|^{2} \int_{K \cap\{|x-y| \geq 1\}} \frac{1}{|x-y|^{3+2 s}} d x d y \\
& +C \int_{K}|u(x)|^{2} \int_{K \cap\{|x-y| \leq 1\}} \frac{1}{|x-y|^{1+2 s}} d x d y,
\end{aligned}
$$

where in the last line we used that

$$
\left|e^{-\mathrm{i}(x-y) \cdot A\left(\frac{x+y}{2}\right)}-1\right|^{2} \leq C|x-y|^{2}, \quad \text { for }|x-y| \leq 1, \quad x, y \in K,
$$

since $A$ is locally bounded. The proof is now complete.

Lemma 3.4. Let $\left\{A_{n}\right\}_{n \in \mathbb{N}}$ be a sequence of uniformly locally bounded functions $A_{n}: \mathbb{R}^{3} \rightarrow \mathbb{R}^{3}$ with locally bounded gradient and, for any $n \in \mathbb{N}, u_{n} \in H_{A_{n}}^{s}\left(\mathbb{R}^{3}, \mathbb{C}\right)$ be such that

$$
\sup _{n \in \mathbb{N}}\left\|u_{n}\right\|_{s, A_{n}}<\infty \text {. }
$$

Then, up to a subsequence, $\left\{u_{n}\right\}_{n \in \mathbb{N}}$ converges strongly to some function $u$ in $L^{q}(K, \mathbb{C})$ for every compact set $K$ and any $q \in[1,6 /(3-2 s))$.

Proof. Arguing as in the proof of Lemma 3.3, the assertion follows by [13, Corollary 7.2].

Lemma 3.5 (Magnetic Sobolev embeddings). The injection

$$
H_{A}^{s}\left(\mathbb{R}^{3}, \mathbb{C}\right) \hookrightarrow L^{p}\left(\mathbb{R}^{3}, \mathbb{C}\right)
$$

is continuous for every $2 \leq p \leq \frac{6}{3-2 s}$. Furthermore, the injection

$$
H_{A}^{s}\left(\mathbb{R}^{3}, \mathbb{C}\right) \hookrightarrow L^{p}(K, \mathbb{C})
$$

is compact for every $1 \leq p<\frac{6}{3-2 s}$ and any compact set $K \subset \mathbb{R}^{3}$.

Proof. By combining Remark 3.2 with the continuous injection $H^{s}\left(\mathbb{R}^{3}\right) \hookrightarrow L^{6 /(3-2 s)}\left(\mathbb{R}^{3}\right.$ ) (see $[13$, Theorem 6.5]) yields

$$
\|u\|_{L^{6 /(3-2 s)\left(\mathbb{R}^{3}\right)}} \leq C\left(\int_{\mathbb{R}^{6}} \frac{\left|e^{-\mathrm{i}(x-y) \cdot A\left(\frac{x+y}{2}\right)} u(x)-u(y)\right|^{2}}{|x-y|^{3+2 s}} d x d y\right)^{1 / 2} \text { for all } u \in H_{A}^{s}\left(\mathbb{R}^{3}, \mathbb{C}\right) .
$$

Whence, by interpolation the first assertion immediately follows. For the compact embedding, taking into account Lemma 3.3, the assertion follows by [13, Corollary 7.2].

Lemma 3.6 (Vanishing). Let $\left\{u_{n}\right\}_{n \in \mathbb{N}}$ be a bounded sequence in $H^{s}\left(\mathbb{R}^{3}\right)$ and assume that, for some $R>0$ and $2 \leq q<\frac{6}{3-2 s}$, there holds

$$
\lim _{n} \sup _{\xi \in \mathbb{R}^{3}} \int_{B(\xi, R)}\left|u_{n}\right|^{q} d x=0 .
$$

Then $u_{n} \rightarrow 0$ in $L^{p}\left(\mathbb{R}^{3}\right)$ for $2<p<\frac{6}{3-2 s}$. 
Proof. See [11, Lemma 2.3].

Lemma 3.7 (Localized Sobolev inequality). Let $\xi \in \mathbb{R}^{3}$ and $R>0$. Then, for $u \in H^{s}\left(B_{R}(\xi)\right.$ ),

$$
\|u\|_{L^{\frac{6}{3-2 s}}\left(B_{R}(\xi)\right)} \leq C(s)\left(\frac{1}{R^{2 s}} \int_{B_{R}(\xi)}|u(x)|^{2} d x+\int_{B_{R}(\xi) \times B_{R}(\xi)} \frac{|u(x)-u(y)|^{2}}{|x-y|^{3+2 s}} d y d x\right)^{1 / 2}
$$

for some constant $C(s)>0$. In particular for every $1 \leq p \leq \frac{6}{3-2 s}$ there holds

$$
\|u\|_{L^{p}\left(B_{R}(\xi)\right)} \leq C(s, R)\|u\|_{H^{s}\left(B_{R}(\xi)\right)}
$$

for some constant $C(s, R)>0$ and all $u \in H^{s}\left(B_{R}(\xi)\right)$.

Proof. See [4, Proposition 2.5] for the first inequality. The second inequality immediately follows.

Lemma 3.8 (Cut-off estimates). Let $u \in H_{A}^{s}\left(\mathbb{R}^{3}, \mathbb{C}\right)$ and $\varphi \in C^{0,1}\left(\mathbb{R}^{3}\right)$ with $0 \leq \varphi \leq 1$. Then, for every pair of measurable sets $E_{1}, E_{2} \subset \mathbb{R}^{3}$, we have

$$
\begin{aligned}
\int_{E_{1} \times E_{2}} \frac{\left|e^{-\mathrm{i}(x-y) \cdot A\left(\frac{x+y}{2}\right)} \varphi(x) u(x)-\varphi(y) u(y)\right|^{2}}{|x-y|^{3+2 s}} d x d y \leq & C \min \left\{\int_{E_{1}}|u|^{2} d x, \int_{E_{2}}|u|^{2} d x\right\} \\
& +C \int_{E_{1} \times E_{2}} \frac{\left|e^{-\mathrm{i}(x-y) \cdot A\left(\frac{x+y}{2}\right)} u(x)-u(y)\right|^{2}}{|x-y|^{3+2 s}} d x d y
\end{aligned}
$$

where $C$ depends on $s$ and on the Lipschitz constant of $\varphi$.

Proof. The proof follows by arguing as in [13, Lemma 5.3], where the case $A=0$ and $E_{1}=E_{2}$ is considered. For the sake of completeness, we show the details. We have

$$
\begin{aligned}
& \int_{E_{1} \times E_{2}} \frac{\left|e^{-\mathrm{i}(x-y) \cdot A\left(\frac{x+y}{2}\right)} \varphi(x) u(x)-\varphi(y) u(y)\right|^{2}}{|x-y|^{3+2 s}} d x d y \\
& \leq C \int_{E_{1} \times E_{2}} \frac{\left|e^{-\mathrm{i}(x-y) \cdot A\left(\frac{x+y}{2}\right)} u(x)-u(y)\right|^{2}}{|x-y|^{3+2 s}} d x d y+C \int_{E_{1} \times E_{2}} \frac{|u(y)|^{2}|\varphi(x)-\varphi(y)|^{2}}{|x-y|^{3+2 s}} d x d y .
\end{aligned}
$$

On the other hand, the second integral splits as

$\int_{E_{2}}|u(y)|^{2} \int_{E_{1} \cap\{|x-y| \leq 1\}} \frac{1}{|x-y|^{1+2 s}} d x d y+\int_{E_{2}}|u(y)|^{2} \int_{E_{1} \cap\{|x-y| \geq 1\}} \frac{1}{|x-y|^{3+2 s}} d x d y \leq C \int_{E_{2}}|u|^{2} d y$.

Analogously, we have

$$
\begin{aligned}
& \int_{E_{1} \times E_{2}} \frac{\left|\varphi(x) u(x)-e^{\mathrm{i}(x-y) \cdot A\left(\frac{x+y}{2}\right)} \varphi(y) u(y)\right|^{2}}{|x-y|^{3+2 s}} d x d y \\
& \leq C \int_{E_{1} \times E_{2}} \frac{\left|e^{-\mathrm{i}(x-y) \cdot A\left(\frac{x+y}{2}\right)} u(x)-u(y)\right|^{2}}{|x-y|^{3+2 s}} d x d y+C \int_{E_{1} \times E_{2}} \frac{|u(x)|^{2}|\varphi(x)-\varphi(y)|^{2}}{|x-y|^{3+2 s}} d x d y,
\end{aligned}
$$

and the second term can be estimated as before by $\int_{E_{1}}|u|^{2} d x$. The assertion follows.

Thus we can prove

Lemma 3.9 (Dicothomy). Let $\left\{u_{n}\right\}_{n \in \mathbb{N}}$ be a sequence in $H_{A}^{s}\left(\mathbb{R}^{3}, \mathbb{C}\right)$ such that, for some $L>0$,

$$
\left\|u_{n}\right\|_{L^{p}\left(\mathbb{R}^{3}\right)}=1, \quad \lim _{n}\left\|u_{n}\right\|_{s, A}^{2}=L,
$$

and let us set

$$
\mu_{n}(x)=\left|u_{n}(x)\right|^{2}+\int_{\mathbb{R}^{3}} \frac{\left|e^{-\mathrm{i}(x-y) \cdot A\left(\frac{x+y}{2}\right)} u_{n}(x)-u_{n}(y)\right|^{2}}{|x-y|^{3+2 s}} d y, \quad x \in \mathbb{R}^{3}, n \in \mathbb{N} .
$$


Assume that there exists $\beta \in(0, L)$ such that for all $\varepsilon>0$ there exist $\bar{R}>0, \bar{n} \geq 1$, a sequence of radii $R_{n} \rightarrow+\infty$ and $\left\{\xi_{n}\right\}_{n \in \mathbb{N}} \subset \mathbb{R}^{3}$ such that for $n \geq \bar{n}$

$$
\begin{aligned}
& \left|\int_{\mathbb{R}^{3}} \mu_{n}^{1}(x) d x-\beta\right| \leq \varepsilon, \quad \mu_{n}^{1}:=1_{B_{\bar{R}}\left(\xi_{n}\right)} \mu_{n}, \\
& \left|\int_{\mathbb{R}^{3}} \mu_{n}^{2}(x) d x-(L-\beta)\right| \leq \varepsilon, \quad \mu_{n}^{2}:=1_{B_{R_{n}}^{c}\left(\xi_{n}\right)} \mu_{n}, \\
& \int_{\mathbb{R}^{3}}\left|\mu_{n}(x)-\mu_{n}^{1}(x)-\mu_{n}^{2}(x)\right| d x \leq \varepsilon .
\end{aligned}
$$

Then there exist $\left\{u_{n}^{1}\right\}_{n \in \mathbb{N}},\left\{u_{n}^{2}\right\}_{n \in \mathbb{N}} \subset H_{A}^{s}\left(\mathbb{R}^{3}, \mathbb{C}\right)$ such that $\operatorname{dist}\left(\operatorname{supp}\left(u_{n}^{1}\right), \operatorname{supp}\left(u_{n}^{2}\right)\right) \rightarrow+\infty$ and

$$
\begin{aligned}
& \left|\left\|u_{n}^{1}\right\|_{s, A}^{2}-\beta\right| \leq \varepsilon, \\
& \left|\left\|u_{n}^{2}\right\|_{s, A}^{2}-(L-\beta)\right| \leq \varepsilon, \\
& \left\|u_{n}-u_{n}^{1}-u_{n}^{2}\right\|_{s, A} \leq \varepsilon, \\
& \left|1-\left\|u_{n}^{1}\right\|_{L^{p}\left(\mathbb{R}^{3}\right)}^{p}-\left\|u_{n}^{2}\right\|_{L^{p}\left(\mathbb{R}^{3}\right)}^{p}\right| \leq \varepsilon
\end{aligned}
$$

for any $n \geq \bar{n}$.

Proof. Notice that we have

$$
\begin{aligned}
\int_{\mathbb{R}^{3}} \mu_{n}^{1} d x= & \int_{B_{\bar{R}}\left(\xi_{n}\right)}\left|u_{n}\right|^{2} d x+\int_{B_{\bar{R}}\left(\xi_{n}\right) \times B_{\bar{R}}\left(\xi_{n}\right)} \frac{\left|e^{-\mathrm{i}(x-y) \cdot A\left(\frac{x+y}{2}\right)} u_{n}(x)-u_{n}(y)\right|^{2}}{|x-y|^{3+2 s}} d x d y \\
& +\int_{B_{\bar{R}}\left(\xi_{n}\right) \times B_{\bar{R}}^{c}\left(\xi_{n}\right)} \frac{\left|e^{-\mathrm{i}(x-y) \cdot A\left(\frac{x+y}{2}\right)} u_{n}(x)-u_{n}(y)\right|^{2}}{|x-y|^{3+2 s}} d x d y
\end{aligned}
$$

as well as

$$
\begin{aligned}
\int_{\mathbb{R}^{3}} \mu_{n}^{2} d x= & \int_{B_{R_{n}}^{c}\left(\xi_{n}\right)}\left|u_{n}\right|^{2} d x+\int_{B_{R_{n}}^{c}\left(\xi_{n}\right) \times B_{R_{n}}^{c}\left(\xi_{n}\right)} \frac{\left|e^{-\mathrm{i}(x-y) \cdot A\left(\frac{x+y}{2}\right)} u_{n}(x)-u_{n}(y)\right|^{2}}{|x-y|^{3+2 s}} d x d y \\
& +\int_{B_{R_{n}}^{c}\left(\xi_{n}\right) \times B_{R_{n}}\left(\xi_{n}\right)} \frac{\left|e^{-\mathrm{i}(x-y) \cdot A\left(\frac{x+y}{2}\right)} u_{n}(x)-u_{n}(y)\right|^{2}}{|x-y|^{3+2 s}} d x d y,
\end{aligned}
$$

and, from inequality (3.2), we have, for $n \geq \bar{n}$,

$$
\begin{aligned}
& \int_{\left\{\bar{R} \leq\left|x-\xi_{n}\right| \leq R_{n}\right\} \times \mathbb{R}^{3}} \frac{\left|e^{-\mathrm{i}(x-y) \cdot A\left(\frac{x+y}{2}\right)} u_{n}(x)-u_{n}(y)\right|^{2}}{|x-y|^{3+2 s}} d x d y \leq \varepsilon, \\
& \int_{\mathbb{R}^{3} \times\left\{\bar{R} \leq\left|y-\xi_{n}\right| \leq R_{n}\right\}} \frac{\left|e^{-\mathrm{i}(x-y) \cdot A\left(\frac{x+y}{2}\right)} u_{n}(x)-u_{n}(y)\right|^{2}}{|x-y|^{3+2 s}} d x d y \leq \varepsilon, \\
& \int_{\left\{\bar{R} \leq\left|x-\xi_{n}\right| \leq R_{n}\right\}}\left|u_{n}\right|^{2} d x \leq \varepsilon .
\end{aligned}
$$

For every $r>0$, let $\varphi_{r} \in C^{\infty}\left(\mathbb{R}^{3}\right)$ be a radially symmetric function such that $\varphi_{r}=1$ on $B_{r}(0)$ and $\varphi_{r}=0 \mathrm{su} B_{2 r}^{c}(0)$. In light of Lemma 3.8 applied with $E_{1}=E_{2}=\mathbb{R}^{3}$, for any $n \in \mathbb{N}$, we can consider the functions

$$
u_{n}^{1}:=\varphi_{\bar{R}}\left(\cdot-\xi_{n}\right) u_{n} \in H_{A}^{s}\left(\mathbb{R}^{3}, \mathbb{C}\right), \quad u_{n}^{2}:=\left(1-\varphi_{R_{n} / 2}\left(\cdot-\xi_{n}\right)\right) u_{n} \in H_{A}^{s}\left(\mathbb{R}^{3}, \mathbb{C}\right) .
$$

We observe for further usage that the functions $\varphi_{\bar{R}}\left(\cdot-\xi_{n}\right)$ and $1-\varphi_{R_{n} / 2}\left(\cdot-\xi_{n}\right)$ have a Lipschitz constant which is uniformly bounded with respect to $n$. Moreover, $\operatorname{dist}\left(\operatorname{supp}\left(u_{n}^{1}\right), \operatorname{supp}\left(u_{n}^{2}\right)\right) \rightarrow \infty$. 
Let us consider $\left\{u_{n}^{1}\right\}_{n \in \mathbb{N}}$. We have $\left[u_{n}^{1}\right]_{s, A}^{2}=\sum_{i=1}^{5} I_{n}^{i}$, where

$$
\begin{aligned}
I_{n}^{1} & :=\int_{B_{\bar{R}}\left(\xi_{n}\right) \times B_{\bar{R}}\left(\xi_{n}\right)} \frac{\left|e^{-\mathrm{i}(x-y) \cdot A\left(\frac{x+y}{2}\right)} u_{n}(x)-u_{n}(y)\right|^{2}}{|x-y|^{3+2 s}} d x d y, \\
I_{n}^{2} & :=\int_{B_{2 \bar{R}}\left(\xi_{n}\right) \backslash B_{\bar{R}}\left(\xi_{n}\right) \times B_{2 \bar{R}}\left(\xi_{n}\right) \backslash B_{\bar{R}}\left(\xi_{n}\right)} \frac{\left|e^{-\mathrm{i}(x-y) \cdot A\left(\frac{x+y}{2}\right)} u_{n}^{1}(x)-u_{n}^{1}(y)\right|^{2}}{|x-y|^{3+2 s}} d x d y, \\
I_{n}^{3} & :=2 \int_{B_{2 \bar{R}}\left(\xi_{n}\right) \backslash B_{\bar{R}}\left(\xi_{n}\right) \times B_{\bar{R}}\left(\xi_{n}\right)} \frac{\left|e^{-\mathrm{i}(x-y) \cdot A\left(\frac{x+y}{2}\right)} u_{n}^{1}(x)-u_{n}^{1}(y)\right|^{2}}{|x-y|^{3+2 s}} d x d y, \\
I_{n}^{4} & :=2 \int_{B_{2 \bar{R}}\left(\xi_{n}\right) \backslash B_{\bar{R}}\left(\xi_{n}\right) \times B_{2 \bar{R}}^{c}\left(\xi_{n}\right)} \frac{\left|e^{-\mathrm{i}(x-y) \cdot A\left(\frac{x+y}{2}\right)} u_{n}^{1}(x)-u_{n}^{1}(y)\right|^{2}}{|x-y|^{3+2 s}} d x d y, \\
I_{n}^{5} & :=2 \int_{B_{\bar{R}}\left(\xi_{n}\right) \times B_{2 \bar{R}}^{c}\left(\xi_{n}\right)} \frac{\left|e^{-\mathrm{i}(x-y) \cdot A\left(\frac{x+y}{2}\right)} u_{n}^{1}(x)-u_{n}^{1}(y)\right|^{2}}{|x-y|^{3+2 s}} d x d y .
\end{aligned}
$$

Concerning $I_{n}^{i}$ with $i=2,3,4$, since for suitable measurable sets $E_{2}^{i} \subset \mathbb{R}^{3}$ and $c_{i}>0$,

$$
I_{n}^{i}=c_{i} \int_{B_{2 \bar{R}}\left(\xi_{n}\right) \backslash B_{\bar{R}}\left(\xi_{n}\right) \times E_{2}^{i}} \frac{\left|e^{-\mathrm{i}(x-y) \cdot A\left(\frac{x+y}{2}\right)} u_{n}^{1}(x)-u_{n}^{1}(y)\right|^{2}}{|x-y|^{3+2 s}} d x d y,
$$

in light of Lemma 3.8 and inequalities (3.8)-(3.10), we have

$$
\begin{aligned}
I_{n}^{i} & \leq C\left[\int_{B_{2 \bar{R}}\left(\xi_{n}\right) \backslash B_{\bar{R}}\left(\xi_{n}\right)}\left|u_{n}\right|^{2} d x+\int_{B_{2 \bar{R}}\left(\xi_{n}\right) \backslash B_{\bar{R}}\left(\xi_{n}\right) \times E_{2}^{i}} \frac{\left|e^{-\mathrm{i}(x-y) \cdot A\left(\frac{x+y}{2}\right)} u_{n}(x)-u_{n}(y)\right|^{2}}{|x-y|^{3+2 s}} d x d y\right] \\
& \leq C \varepsilon
\end{aligned}
$$

being $B_{2 \bar{R}}\left(\xi_{n}\right) \backslash B_{\bar{R}}\left(\xi_{n}\right) \subset\left\{\bar{R} \leq\left|x-\xi_{n}\right| \leq R_{n}\right\}$ for every $n$ large enough.

Concerning $I_{n}^{5}$, we have

$$
\begin{aligned}
I_{n}^{5}= & 2 \int_{B_{\bar{R}}\left(\xi_{n}\right) \times\left\{2 \bar{R} \leq\left|y-\xi_{n}\right| \leq R_{n}\right\}} \frac{\left|e^{-\mathrm{i}(x-y) \cdot A\left(\frac{x+y}{2}\right)} u_{n}^{1}(x)-u_{n}^{1}(y)\right|^{2}}{|x-y|^{3+2 s}} d x d y \\
& +2 \int_{B_{\bar{R}}\left(\xi_{n}\right) \times B_{R_{n}}^{c}\left(\xi_{n}\right)} \frac{\left|e^{-\mathrm{i}(x-y) \cdot A\left(\frac{x+y}{2}\right)} u_{n}^{1}(x)-u_{n}^{1}(y)\right|^{2}}{|x-y|^{3+2 s}} d x d y .
\end{aligned}
$$

Then, arguing as in (3.11) for $I_{n}^{i}(i=2,3,4)$ we get

$$
\int_{B_{\bar{R}}\left(\xi_{n}\right) \times\left\{2 \bar{R} \leq\left|y-\xi_{n}\right| \leq R_{n}\right\}} \frac{\left|e^{-\mathrm{i}(x-y) \cdot A\left(\frac{x+y}{2}\right)} u_{n}^{1}(x)-u_{n}^{1}(y)\right|^{2}}{|x-y|^{3+2 s}} d x d y \leq C \varepsilon,
$$

for large $n$. On the other hand, as far as the second term in concerned, we get

$$
\int_{B_{\bar{R}}\left(\xi_{n}\right) \times B_{R_{n}}^{c}\left(\xi_{n}\right)} \frac{\left|e^{-\mathrm{i}(x-y) \cdot A\left(\frac{x+y}{2}\right)} u_{n}^{1}(x)-u_{n}^{1}(y)\right|^{2}}{|x-y|^{3+2 s}} d x d y=\int_{B_{\bar{R}}\left(\xi_{n}\right) \times B_{R_{n}}^{c}\left(\xi_{n}\right)} \frac{\left|u_{n}(x)\right|^{2}}{|x-y|^{3+2 s}} d x d y,
$$


since $u_{n}^{1}(y)=0$ for all $y \in B_{R_{n}}^{c}\left(\xi_{n}\right)$ and $u_{n}^{1}(x)=u_{n}(x)$ for all $x \in B_{\bar{R}}\left(\xi_{n}\right)$. Observe first that if $(x, y) \in B_{\bar{R}}\left(\xi_{n}\right) \times B_{R_{n}}^{c}\left(\xi_{n}\right)$, then $|x-y| \geq R_{n}-\bar{R} \rightarrow \infty$, as $n \rightarrow \infty$. We thus have

$$
\begin{aligned}
& \int_{B_{\bar{R}}\left(\xi_{n}\right) \times B_{R_{n}}^{c}\left(\xi_{n}\right)} \frac{\left|u_{n}(x)\right|^{2}}{|x-y|^{3+2 s}} d x d y \\
& \leq \frac{1}{\left(R_{n}-\bar{R}\right)^{\delta}} \int_{B_{\bar{R}}\left(\xi_{n}\right)}\left|u_{n}(x)\right|^{2}\left(\int_{\{|x-y| \geq 1\}} \frac{1}{|x-y|^{3+2 s-\delta}} d y\right) d x \leq \frac{C}{\left(R_{n}-\bar{R}\right)^{\delta}} \leq C \varepsilon,
\end{aligned}
$$

where $0<\delta<2 s$. Here we have used the boundedness of $\left\{u_{n}\right\}_{n \in \mathbb{N}}$ in $L^{2}\left(\mathbb{R}^{3}, \mathbb{C}\right)$. So we have that $\left[u_{n}^{1}\right]_{s, A}^{2}=I_{n}^{1}+\varsigma_{n, \varepsilon}$ with $\varsigma_{n, \varepsilon} \leq C \varepsilon$ for $n$ large, which implies on account of (3.10)

$$
\left\|u_{n}^{1}\right\|_{s, A}^{2}=\int_{B_{\bar{R}}\left(\xi_{n}\right)}\left|u_{n}\right|^{2} d x+I_{n}^{1}+\varsigma_{n, \varepsilon}, \quad \varsigma_{n, \varepsilon} \leq C \varepsilon .
$$

A similar argument involving $\left\{u_{n}\right\}_{n \in \mathbb{N}}$ in place of $\left\{u_{n}^{1}\right\}_{n \in \mathbb{N}}$ shows that formula (3.7) writes as

$$
\int_{\mathbb{R}^{3}} \mu_{n}^{1} d x=\int_{B_{\bar{R}}\left(\xi_{n}\right)}\left|u_{n}\right|^{2} d x+I_{n}^{1}+\hat{\varsigma}_{n, \varepsilon}, \quad \hat{\varsigma}_{n, \varepsilon} \leq C \varepsilon
$$

Indeed, since

$$
\begin{aligned}
& \int_{B_{\bar{R}}\left(\xi_{n}\right) \times B_{\bar{R}}^{c}\left(\xi_{n}\right)} \frac{\left|e^{-\mathrm{i}(x-y) \cdot A\left(\frac{x+y}{2}\right)} u_{n}(x)-u_{n}(y)\right|^{2}}{|x-y|^{3+2 s}} d x d y \\
& \leq \int_{B_{\bar{R}}\left(\xi_{n}\right) \times\left\{\bar{R} \leq\left|y-\xi_{n}\right| \leq R_{n}\right\}} \frac{\left|e^{-\mathrm{i}(x-y) \cdot A\left(\frac{x+y}{2}\right)} u_{n}(x)-u_{n}(y)\right|^{2}}{|x-y|^{3+2 s}} d x d y \\
& \quad+C\left[\int_{B_{\bar{R}}\left(\xi_{n}\right) \times B_{R_{n}}^{c}\left(\xi_{n}\right)} \frac{\left|u_{n}(x)\right|^{2}}{|x-y|^{3+2 s}} d x d y+\int_{B_{\bar{R}}\left(\xi_{n}\right) \times B_{R_{n}}^{c}\left(\xi_{n}\right)} \frac{\left|u_{n}(y)\right|^{2}}{|x-y|^{3+2 s}} d x d y\right]
\end{aligned}
$$

by (3.9) and arguing as in (3.12) we can conclude. By combining (3.13) and (3.14) we finally obtain the desired estimate (3.3).

Now, concerning $\left\{u_{n}^{2}\right\}_{n \in \mathbb{N}}$, we have $\left[u_{n}^{2}\right]_{s, A}^{2}=\sum_{i=1}^{5} J_{n}^{i}$, where we have set

$$
\begin{aligned}
& J_{n}^{1}:=\int_{B_{R_{n}}^{c}\left(\xi_{n}\right) \times B_{R_{n}}^{c}\left(\xi_{n}\right)} \frac{\left|e^{-\mathrm{i}(x-y) \cdot A\left(\frac{x+y}{2}\right)} u_{n}(x)-u_{n}(y)\right|^{2}}{|x-y|^{3+2 s}} d x d y, \\
& J_{n}^{2}:=\int_{B_{R_{n}}\left(\xi_{n}\right) \backslash B_{R_{n} / 2}\left(\xi_{n}\right) \times B_{R_{n}}\left(\xi_{n}\right) \backslash B_{R_{n} / 2}\left(\xi_{n}\right)} \frac{\left|e^{-\mathrm{i}(x-y) \cdot A\left(\frac{x+y}{2}\right)} u_{n}^{2}(x)-u_{n}^{2}(y)\right|^{2}}{|x-y|^{3+2 s}} d x d y, \\
& J_{n}^{3}:=2 \int_{B_{R_{n}}\left(\xi_{n}\right) \backslash B_{R_{n} / 2}\left(\xi_{n}\right) \times B_{R_{n} / 2}\left(\xi_{n}\right)} \frac{\left|e^{-\mathrm{i}(x-y) \cdot A\left(\frac{x+y}{2}\right)} u_{n}^{2}(x)-u_{n}^{2}(y)\right|^{2}}{|x-y|^{3+2 s}} d x d y, \\
& J_{n}^{4}:=2 \int_{B_{R_{n}}\left(\xi_{n}\right) \backslash B_{R_{n} / 2}\left(\xi_{n}\right) \times B_{R_{n}}^{c}\left(\xi_{n}\right)} \frac{\left|e^{-\mathrm{i}(x-y) \cdot A\left(\frac{x+y}{2}\right)} u_{n}^{2}(x)-u_{n}^{2}(y)\right|^{2}}{|x-y|^{3+2 s}} d x d y, \\
& J_{n}^{5}:=2 \int_{B_{R_{n} / 2}\left(\xi_{n}\right) \times B_{R_{n}}^{c}\left(\xi_{n}\right)} \frac{\left|e^{-\mathrm{i}(x-y) \cdot A\left(\frac{x+y}{2}\right)} u_{n}^{2}(x)-u_{n}^{2}(y)\right|^{2}}{|x-y|^{3+2 s}} d x d y .
\end{aligned}
$$

Concerning $J_{n}^{i}$ with $i=2,3,4$, observe that the integration domains are $B_{R_{n}}\left(\xi_{n}\right) \backslash B_{R_{n} / 2}\left(\xi_{n}\right) \times E_{2}^{i}$, for suitable measurable $E_{2}^{i}$, s, and they are subset of $\left\{\bar{R} \leq\left|x-\xi_{n}\right| \leq R_{n}\right\} \times \mathbb{R}^{3}$ for $n$ sufficiently 
large. Thus we can argue as in (3.11). Finally, $J_{n}^{5}$ can be estimated with similar arguments to that used in (3.12) and, as for $\mu_{n}^{1}$, using also (3.10), we obtain

$$
\int_{\mathbb{R}^{3}} \mu_{n}^{2}(x) d x=\int_{B_{R_{n}}^{c}\left(\xi_{n}\right)}\left|u_{n}\right|^{2} d x+J_{n}^{1}+\bar{\varsigma}_{n, \varepsilon}, \quad \bar{\varsigma}_{n, \varepsilon} \leq C \varepsilon .
$$

By combining all these estimates we get (3.4) for any $n$ large.

Conclusion (3.5) follows by (3.8)-(3.10). In fact, setting

$$
v_{n}:=u_{n}-u_{n}^{1}-u_{n}^{2}=\left(\varphi_{R_{n} / 2}\left(\cdot-\xi_{n}\right)-\varphi_{\bar{R}}\left(\cdot-\xi_{n}\right)\right) u_{n}
$$

for all $n$, inequality (3.10) yields

$$
\int_{\mathbb{R}^{3}}\left|v_{n}\right|^{2} d x=\int_{\mathbb{R}^{3}}\left(\varphi_{R_{n} / 2}\left(x-\xi_{n}\right)-\varphi_{\bar{R}}\left(x-\xi_{n}\right)\right)^{2}\left|u_{n}\right|^{2} d x \leq \int_{\left\{\bar{R} \leq\left|x-\xi_{n}\right| \leq R_{n}\right\}}\left|u_{n}\right|^{2} d x \leq \varepsilon .
$$

Furthermore, $\left[v_{n}\right]_{s, A}^{2}=\sum_{i=1}^{4} K_{n}^{i}$, where

$$
\begin{aligned}
& K_{n}^{1}:=\int_{B_{R_{n}}\left(\xi_{n}\right) \backslash B_{\bar{R}}\left(\xi_{n}\right) \times B_{R_{n}}\left(\xi_{n}\right) \backslash B_{\bar{R}}\left(\xi_{n}\right)} \frac{\left|e^{-\mathrm{i}(x-y) \cdot A\left(\frac{x+y}{2}\right)} v_{n}(x)-v_{n}(y)\right|^{2}}{|x-y|^{3+2 s}} d x d y, \\
& K_{n}^{2}:=2 \int_{B_{R_{n}}\left(\xi_{n}\right) \backslash B_{\bar{R}}\left(\xi_{n}\right) \times B_{\bar{R}}\left(\xi_{n}\right)} \frac{\left|e^{-\mathrm{i}(x-y) \cdot A\left(\frac{x+y}{2}\right)} v_{n}(x)-v_{n}(y)\right|^{2}}{|x-y|^{3+2 s}} d x d y, \\
& K_{n}^{3}:=2 \int_{B_{R_{n}}\left(\xi_{n}\right) \backslash B_{\bar{R}}\left(\xi_{n}\right) \times B_{R_{n}}^{c}\left(\xi_{n}\right)} \frac{\left|e^{-\mathrm{i}(x-y) \cdot A\left(\frac{x+y}{2}\right)} v_{n}(x)-v_{n}(y)\right|^{2}}{|x-y|^{3+2 s}} d x d y .
\end{aligned}
$$

Since $v_{n}=\tilde{\varphi} u_{n}$ with $\tilde{\varphi}:=\left(\varphi_{R_{n} / 2}\left(\cdot-\xi_{n}\right)-\varphi_{\bar{R}}\left(\cdot-\xi_{n}\right)\right)$, we can repeat the arguments performed in (3.11). Concerning the final assertion (3.6), we have for some $\vartheta>0$,

$$
\begin{aligned}
1-\left\|u_{n}^{1}\right\|_{L^{p}}^{p}-\left\|u_{n}^{2}\right\|_{L^{p}}^{p} & =\int_{\mathbb{R}^{3}}\left(1-\varphi_{\bar{R}}^{p}\left(x-\xi_{n}\right)-\left(1-\varphi_{R_{n} / 2}\left(x-\xi_{n}\right)\right)^{p}\right)\left|u_{n}\right|^{p} d x \\
& \leq \int_{\left\{\bar{R} \leq\left|x-\xi_{n}\right| \leq R_{n}\right\}}\left|u_{n}\right|^{p} d x \\
& \leq\left(\int_{\left\{\bar{R} \leq\left|x-\xi_{n}\right| \leq R_{n}\right\}}\left|u_{n}\right|^{2} d x\right)^{\frac{\vartheta p}{2}}\left(\int_{\mathbb{R}^{3}}\left|u_{n}\right|^{\frac{6}{3-2 s}} d x\right)^{\frac{(1-\vartheta) p(3-2 s)}{6}} \\
& \leq \varepsilon
\end{aligned}
$$

in light of (3.10) and Lemma 3.5. This concludes the proof.

Lemma 3.10 (Partial Gauge invariance). Let $\xi \in \mathbb{R}^{3}$ and $u \in H_{A}^{s}\left(\mathbb{R}^{3}, \mathbb{C}\right)$. For $\eta \in \mathbb{R}^{3}$, let us set

$$
v(x)=e^{\mathrm{i} \eta \cdot x} u(x+\xi), \quad x \in \mathbb{R}^{3} .
$$

Then $v \in H_{A_{\eta}}^{s}\left(\mathbb{R}^{3}, \mathbb{C}\right)$ and

$$
\|u\|_{s, A}=\|v\|_{s, A_{\eta}}, \quad \text { where } A_{\eta}:=A(\cdot+\xi)+\eta .
$$

Proof. Of course $\|v\|_{L^{2}}=\|u\|_{L^{2}}$. Moreover, a change of variables yields

$$
\begin{aligned}
\int_{\mathbb{R}^{6}} \frac{\left|e^{-\mathrm{i}(x-y) \cdot A_{\eta}\left(\frac{x+y}{2}\right)} v(x)-v(y)\right|^{2}}{|x-y|^{3+2 s}} d x d y & =\int_{\mathbb{R}^{6}} \frac{\left|e^{-\mathrm{i}(x-y) \cdot A\left(\frac{x+y}{2}+\xi\right)} u(x+\xi)-u(y+\xi)\right|^{2}}{|x-y|^{3+2 s}} d x d y \\
& =\int_{\mathbb{R}^{6}} \frac{\left|e^{-\mathrm{i}(x-y) \cdot A\left(\frac{x+y}{2}\right)} u(x)-u(y)\right|^{2}}{|x-y|^{3+2 s}} d x d y,
\end{aligned}
$$

which yields the assertion. 
If $A$ is linear, then, taking $\eta=-A(\xi)$ in Lemma 3.10, we get $A_{\eta}=A$ and hence

Lemma 3.11 (Partial Gauge invariance). Let $\xi \in \mathbb{R}^{3}$ and $u \in H_{A}^{s}\left(\mathbb{R}^{3}, \mathbb{C}\right)$. Assume that $A$ is linear and let us set

$$
v(x)=e^{-\mathrm{i} A(\xi) \cdot x} u(x+\xi), \quad x \in \mathbb{R}^{3} .
$$

Then $v \in H_{A}^{s}\left(\mathbb{R}^{3}, \mathbb{C}\right)$ and $\|u\|_{s, A}=\|v\|_{s, A}$.

\section{EXISTENCE OF MiNIMIZERS}

Let $2<p<6 /(3-2 s)$ and consider the minimization problem $\left(\mathscr{M}_{A}\right)$. First of all observe that by Sobolev embedding, $\mathscr{M}_{A}>0$. Once a solution to $\left(\mathscr{M}_{A}\right)$ exists, due to the Lagrange Multiplier Theorem, there is $\lambda \in \mathbb{R}$ such that

$$
\begin{aligned}
& \frac{c_{s}}{2} \Re \int_{\mathbb{R}^{6}} \frac{\left(e^{-\mathrm{i}(x-y) \cdot A\left(\frac{x+y}{2}\right)} u(x)-u(y)\right) \overline{\left(e^{-\mathrm{i}(x-y) \cdot A\left(\frac{x+y}{2}\right)} v(x)-v(y)\right)}}{|x-y|^{3+2 s}} d x d y \\
& +\Re \int_{\mathbb{R}^{3}} u \bar{v} d x=\lambda \Re \int_{\mathbb{R}^{3}}|u|^{p-2} u \bar{v} d x, \quad \text { for all } v \in H_{A}^{s}\left(\mathbb{R}^{3}, \mathbb{C}\right) .
\end{aligned}
$$

A multiple of $u$ removes the Lagrange multiplier $\lambda$ and provides a weak solution to $\left(\mathscr{P}_{s, A}\right)$. Moreover, if we set

$$
\mathscr{M}_{A}(\lambda):=\inf _{u \in \mathscr{S}(\lambda)}\|u\|_{s, A}^{2}
$$

where

$$
\mathscr{S}(\lambda):=\left\{u \in H_{A}^{s}\left(\mathbb{R}^{3}, \mathbb{C}\right): \int_{\mathbb{R}^{3}}|u|^{p} d x=\lambda\right\},
$$

we have that for every $\lambda>0$

$$
\mathscr{M}_{A}(\lambda)=\lambda^{\frac{2}{p}} \mathscr{M}_{A}
$$

4.1. Subcritical symmetric case. Let $2<p<\frac{6}{3-2 s}$ and consider the problem

$$
\mathscr{M}_{A, r}=\inf _{u \in \mathscr{S}_{r}}\|u\|_{s, A}^{2} \text {, }
$$

where

$$
\mathscr{S}_{r}=\left\{u \in H_{A, \operatorname{rad}}^{s}\left(\mathbb{R}^{3}, \mathbb{C}\right): \int_{\mathbb{R}^{3}}|u|^{p} d x=1\right\} .
$$

First we give the following preliminary result.

Lemma 4.1 (Compact radial embedding). For every $2<q<6 /(3-2 s)$, the mapping

$$
H_{A, \operatorname{rad}}^{s}\left(\mathbb{R}^{3}, \mathbb{C}\right) \ni u \mapsto|u| \in L^{q}\left(\mathbb{R}^{3}\right),
$$

is compact.

Proof. By Lemma 3.1, namely the Diamagnetic inequality, we know that the mapping

$$
H_{A}^{s}\left(\mathbb{R}^{3}, \mathbb{C}\right) \ni u \mapsto|u| \in H^{s}\left(\mathbb{R}^{3}, \mathbb{R}\right),
$$

is continuous. Then, the assertion follows directly by [24, Theorem II.1].

We are ready to prove (i) of Theorem 1.2

Theorem 4.2 (Existence of radial minimizers). For any $2<p<6 /(3-2 s)$, the minimization problem $\mathscr{M}_{A, r}$ admits a solution. In particular, there exists a nontrivial radially symmetric weak solution $u \in H_{A, \text { rad }}^{s}\left(\mathbb{R}^{3}, \mathbb{C}\right)$ to the problem $\left(\mathscr{P}_{s, A}\right)$. 
Proof. Let $\left\{u_{n}\right\}_{n \in \mathbb{N}} \subset \mathscr{S}_{r}$ be a minimizing sequence for $\mathscr{M}_{A, r}$, namely $\left\|u_{n}\right\|_{L^{p}\left(\mathbb{R}^{3}\right)}=1$ for all $n$ and $\left\|u_{n}\right\|_{s, A}^{2} \rightarrow \mathscr{M}_{A, r}$, as $n \rightarrow \infty$. Then, up to a subsequence, it converges weakly to some radial function $u$. On account of Lemma 3.5, $u_{n} \rightarrow u$ a.e. up to a subsequence. By Lemma 4.1, up to a subsequence $\left\{\left|u_{n}\right|\right\}_{n \in \mathbb{N}}$ converges strongly to some $v$ in $L^{q}\left(\mathbb{R}^{3}\right)$ for every $2<q<6 /(3-2 s)$. Of course, $v=|u|$ by pointwise convergence. In particular we can pass to the limit into the constraint $\left\|u_{n}\right\|_{L^{p}\left(\mathbb{R}^{3}\right)}=1$ to get $\|u\|_{L^{p}\left(\mathbb{R}^{3}\right)}=1$. Then $u$ is a solution to $\mathscr{M}_{A, r}$, since by virtue of Fatou Lemma

$$
\begin{aligned}
\mathscr{M}_{A, r} & \leq \int_{\mathbb{R}^{3}}|u(x)|^{2} d x+\int_{\mathbb{R}^{6}} \frac{\left|e^{-\mathrm{i}(x-y) \cdot A\left(\frac{x+y}{2}\right)} u(x)-u(y)\right|^{2}}{|x-y|^{3+2 s}} d x d y \\
& \leq \liminf _{n}\left(\int_{\mathbb{R}^{3}}\left|u_{n}(x)\right|^{2} d x+\int_{\mathbb{R}^{6}} \frac{\left|e^{-\mathrm{i}(x-y) \cdot A\left(\frac{x+y}{2}\right)} u_{n}(x)-u_{n}(y)\right|^{2}}{|x-y|^{3+2 s}} d x d y\right)=\mathscr{M}_{A, r} .
\end{aligned}
$$

This concludes the proof.

4.2. Subcritical case. In this subsection we study the minimization problem $\left(\mathscr{M}_{A}\right)$ in the case $2<p<\frac{6}{3-2 s}$.

4.2.1. Constant magnetic field case. Let us consider $\left(\mathscr{M}_{A}\right)$ under the assumption that $A: \mathbb{R}^{3} \rightarrow$ $\mathbb{R}^{3}$ is linear. The local case was extensively studied in [15] for the magnetic potential

$$
A\left(x_{1}, x_{2}, x_{3}\right)=\frac{b}{2}\left(-x_{2}, x_{1}, 0\right), \quad b \in \mathbb{R} \backslash\{0\} .
$$

Hence we can prove (ii) of Theorem 1.2.

Theorem 4.3 (Existence of minimizers, I). Assume that the potential $A: \mathbb{R}^{3} \rightarrow \mathbb{R}^{3}$ is linear. Then, for any $2<p<\frac{6}{3-2 s}$ the minimization problem $\left(\mathscr{M}_{A}\right)$ admits a solution.

Proof. Let $\left\{u_{n}\right\}_{n \in \mathbb{N}} \subset \mathscr{S}$ be a minimizing sequence for $\mathscr{M}_{A}$, namely $\left\|u_{n}\right\|_{L^{p}}=1$ for all $n$ and $\left\|u_{n}\right\|_{s, A}^{2} \rightarrow \mathscr{M}_{A}$, as $n \rightarrow \infty$. We want to develop a concentration compactness argument [25] on the measure of density defined by

$$
\mu_{n}(x):=\left|u_{n}(x)\right|^{2}+\int_{\mathbb{R}^{3}} \frac{\left|e^{-\mathrm{i}(x-y) \cdot A\left(\frac{x+y}{2}\right)} u_{n}(x)-u_{n}(y)\right|^{2}}{|x-y|^{3+2 s}} d y, \quad x \in \mathbb{R}^{3}, n \in \mathbb{N} .
$$

Notice that $\left\{\mu_{n}\right\}_{n \in \mathbb{N}} \subset L^{1}\left(\mathbb{R}^{3}\right)$ and, since $\left\|u_{n}\right\|_{s, A}^{2}=\mathscr{M}_{A}+o_{n}(1)$,

$$
\sup _{n \in \mathbb{N}} \int_{\mathbb{R}^{3}} \mu_{n}(x) d x<\infty .
$$

More precisely, we shall apply [25, Lemma I.1] by taking $\rho_{n}=\mu_{n}$. Only vanishing, dichotomy or tightness (yielding compactness) are possible. Vanishing can be ruled out. In fact, assume by contradiction that, for all $R>0$ fixed, there holds

$$
\lim _{n} \sup _{\xi \in \mathbb{R}^{N}} \int_{B_{R}(\xi)} \mu_{n}(x) d x=0,
$$

namely

$$
\lim _{n} \sup _{\xi \in \mathbb{R}^{N}}\left(\int_{B_{R}(\xi)}\left|u_{n}(x)\right|^{2} d x+\int_{B_{R}(\xi) \times \mathbb{R}^{3}} \frac{\left|e^{-\mathrm{i}(x-y) \cdot A\left(\frac{x+y}{2}\right)} u_{n}(x)-u_{n}(y)\right|^{2}}{|x-y|^{3+2 s}} d x d y\right)=0 .
$$

By Remark 3.2 it follows that

$$
\lim _{n} \sup _{\xi \in \mathbb{R}^{N}}\left(\int_{B_{R}(\xi)}\left|u_{n}(x)\right|^{2} d x+\int_{B_{R}(\xi) \times \mathbb{R}^{3}} \frac{\|\left. u_{n}(x)|-| u_{n}(y)\right|^{2}}{|x-y|^{3+2 s}} d x d y\right)=0 .
$$


In particular, we get

$$
\lim _{n} \sup _{\xi \in \mathbb{R}^{N}}\left\|\left|u_{n}\right|\right\|_{H^{s}\left(B_{R}(\xi)\right)}^{2}=0
$$

and this implies, by virtue of Lemma 3.7, that for any $R>0$

$$
\lim _{n} \sup _{\xi \in \mathbb{R}^{N}} \int_{B_{R}(\xi)}\left|u_{n}(x)\right|^{p} d x=0 .
$$

Thus, in light of Lemma 3.6, $u_{n} \rightarrow 0$ in $L^{p}$ which violates the constraint $\left\|u_{n}\right\|_{L^{p}}=1$. Whence, vanishing cannot occur.

We now exclude the dicothomy. According to [25, Lemma I.1], this, precisely, means that there exists $\beta \in\left(0, \mathscr{M}_{A}\right)$ such that for all $\varepsilon>0$ there are $\bar{R}>0, \bar{n} \geq 1$, a sequence of radii $R_{n} \rightarrow+\infty$ and $\left\{\xi_{n}\right\}_{n \in \mathbb{N}} \subset \mathbb{R}^{3}$ such that for $n \geq \bar{n}$

$$
\begin{aligned}
& \left|\int_{\mathbb{R}^{3}} \mu_{n}^{1}(x) d x-\beta\right| \leq \varepsilon, \quad \mu_{n}^{1}(x):=1_{B_{\bar{R}}\left(\xi_{n}\right)} \mu_{n}, \\
& \left|\int_{\mathbb{R}^{3}} \mu_{n}^{2}(x) d x-\left(\mathscr{M}_{A}-\beta\right)\right| \leq \varepsilon, \quad \mu_{n}^{2}(x):=1_{B_{R_{n}}^{c}\left(\xi_{n}\right)} \mu_{n}, \\
& \int_{\mathbb{R}^{3}}\left|\mu_{n}(x)-\mu_{n}^{1}(x)-\mu_{n}^{2}(x)\right| d x \leq \varepsilon .
\end{aligned}
$$

Then, by virtue of Lemma 3.9, there exist two sequences $\left\{u_{n}^{1}\right\}_{n \in \mathbb{N}},\left\{u_{n}^{2}\right\}_{n \in \mathbb{N}} \subset H_{A}^{s}\left(\mathbb{R}^{3}, \mathbb{C}\right)$ such that $\operatorname{dist}\left(\operatorname{supp}\left(u_{n}^{1}\right), \operatorname{supp}\left(u_{n}^{2}\right)\right) \rightarrow+\infty$ and

$$
\begin{aligned}
& \left|\left\|u_{n}^{1}\right\|_{s, A}^{2}-\beta\right| \leq \varepsilon \\
& \left|\left\|u_{n}^{2}\right\|_{s, A}^{2}-\left(\mathscr{M}_{A}-\beta\right)\right| \leq \varepsilon \\
& \left|1-\left\|u_{n}^{1}\right\|_{L^{p}}^{p}-\left\|u_{n}^{2}\right\|_{L^{p}}^{p}\right| \leq \varepsilon,
\end{aligned}
$$

for any $n \geq \bar{n}$. Up to a subsequence, in view of $(4.4)$, there exist $\vartheta_{\varepsilon}, \omega_{\varepsilon} \in(0,1)$ such that

$$
\left\|u_{n}^{1}\right\|_{L^{p}}^{p}=: \vartheta_{n, \varepsilon} \rightarrow \vartheta_{\varepsilon}, \quad\left\|u_{n}^{2}\right\|_{L^{p}}^{p}=: \omega_{n, \varepsilon} \rightarrow \omega_{\varepsilon}, \quad\left|1-\vartheta_{\varepsilon}-\omega_{\varepsilon}\right| \leq \varepsilon, \quad \text { as } n \rightarrow \infty .
$$

Notice that $\vartheta_{\varepsilon}$ does not converge to 1 as $\varepsilon \rightarrow 0$, otherwise by (4.1) and (4.2), for $\varepsilon$ small we get

$$
\beta+\varepsilon \geq \limsup _{n}\left\|u_{n}^{1}\right\|_{s, A}^{2} \geq \limsup _{n} \mathscr{M}_{A}\left(\vartheta_{n, \varepsilon}\right)=\mathscr{M}_{A} \vartheta_{\varepsilon}^{2 / p}>\beta+\varepsilon .
$$

Of course $\vartheta_{\varepsilon}$ does not converge to 0 either, as $\varepsilon \rightarrow 0$, otherwise $\omega_{\varepsilon} \rightarrow 1$ and a contradiction would again follow by arguing as above on $u_{n}^{2}$ and using (4.3). Whence, by means of (4.1), (4.2), (4.3), and since $\lambda^{2 / p}+(1-\lambda)^{2 / p}>1$ for any $\lambda \in(0,1)$, if $\varepsilon$ is small enough

$$
\begin{aligned}
& \mathscr{M}_{A}+2 \varepsilon \geq \underset{n}{\limsup }\left(\left\|u_{n}^{1}\right\|_{s, A}^{2}+\left\|u_{n}^{2}\right\|_{s, A}^{2}\right) \geq \underset{n}{\limsup }\left(\mathscr{M}_{A}\left(\vartheta_{n, \varepsilon}\right)+\mathscr{M}_{A}\left(\omega_{n, \varepsilon}\right)\right) \\
& =\mathscr{M}_{A}\left(\vartheta_{\varepsilon}^{2 / p}+\omega_{\varepsilon}^{2 / p}\right)>\mathscr{M}_{A}+2 \varepsilon,
\end{aligned}
$$

a contradiction. This means that tightness needs to occur, namely there exists a sequence $\left\{\xi_{n}\right\}_{n \in \mathbb{N}}$ such that for all $\varepsilon>0$ there exists $R>0$ with

$$
\int_{B_{R}^{c}\left(\xi_{n}\right)}\left|u_{n}(x)\right|^{2} d x+\int_{B_{R}^{c}\left(\xi_{n}\right) \times \mathbb{R}^{3}} \frac{\left|e^{-\mathrm{i}(x-y) \cdot A\left(\frac{x+y}{2}\right)} u_{n}(x)-u_{n}(y)\right|^{2}}{|x-y|^{3+2 s}} d x d y<\varepsilon
$$

for any $n$. In particular, setting $\bar{u}_{n}(x):=u_{n}\left(x+\xi_{n}\right)$, for all $\varepsilon>0$ there is $R>0$ such that

$$
\sup _{n \in \mathbb{N}} \int_{B_{R}^{c}(0)}\left|\bar{u}_{n}(x)\right|^{2} d x<\varepsilon \text {. }
$$


Let us consider

$$
v_{n}(x):=e^{-\mathrm{i} A\left(\xi_{n}\right) \cdot x} \bar{u}_{n}(x), \quad x \in \mathbb{R}^{3} .
$$

Since, by Lemma 3.11, $\left\|v_{n}\right\|_{s, A}=\left\|u_{n}\right\|_{s, A}$, we have that $\left\{v_{n}\right\}_{n \in \mathbb{N}}$ is bounded in $H_{A}^{s}\left(\mathbb{R}^{3}, \mathbb{C}\right)$. Notice also that, since $\left|v_{n}(x)\right|=\left|\bar{u}_{n}(x)\right|$ for a.e. $x \in \mathbb{R}^{3}$ and any $n \in \mathbb{N}$, by (4.5) we have that for all $\varepsilon>0$ there is $R>0$ such that

$$
\sup _{n \in \mathbb{N}} \int_{B_{R}^{c}(0)}\left|v_{n}(x)\right|^{2} d x<\varepsilon .
$$

Thus, in view of the compact injection provided by Lemma 3.5, up to a subsequence, $\left\{v_{n}\right\}_{n \in \mathbb{N}}$ converges weakly, strongly in $L^{2}\left(B_{R}(0), \mathbb{C}\right)$ and point-wisely to some function $v$. Moreover, by (4.6), it follows that $v_{n} \rightarrow v$ strongly in $L^{2}\left(\mathbb{R}^{3}, \mathbb{C}\right)$ as well as in $L^{q}\left(\mathbb{R}^{3}, \mathbb{C}\right)$ for any $2<q<6 /(3-2 s)$, via interpolation. Hence $\|v\|_{L^{p}}=1$. Hence, by Fatou's lemma, we have

$$
\mathscr{M}_{A} \leq\|v\|_{s, A}^{2} \leq \liminf _{n}\left\|v_{n}\right\|_{s, A}^{2}=\liminf _{n}\left\|u_{n}\right\|_{s, A}^{2}=\mathscr{M}_{A},
$$

which proves the existence of a minimizer.

4.2.2. Variable magnetic field case. We now prove (iii) of Theorem 1.2.

Theorem 4.4 (Existence of minimizers, II). Assume that the potential $A: \mathbb{R}^{3} \rightarrow \mathbb{R}^{3}$ satisfies assumption $\mathscr{A}$ and that

$$
\mathscr{M}_{A}<\inf _{\Xi \in \mathscr{X}} \mathscr{M}_{A_{\Xi}} \cdot
$$

Then, for any $2<p<\frac{6}{3-2 s}$, the minimization problem $\left(\mathscr{M}_{A}\right)$ admits a solution.

Proof. By arguing as in the proof of Theorem 4.3, if $\left\{u_{n}\right\}_{n \in \mathbb{N}}$ is a minimizing sequence for $\mathscr{M}_{A}$, we can find a sequence $\left\{\xi_{n}\right\}_{n \in \mathbb{N}}$ such that for all $\varepsilon>0$ there exists $R>0$ with

$$
\int_{B_{R}^{c}\left(\xi_{n}\right)}\left|u_{n}(x)\right|^{2} d x+\int_{B_{R}^{c}\left(\xi_{n}\right) \times \mathbb{R}^{3}} \frac{\left|e^{-\mathrm{i}(x-y) \cdot A\left(\frac{x+y}{2}\right)} u_{n}(x)-u_{n}(y)\right|^{2}}{|x-y|^{3+2 s}} d x d y<\varepsilon
$$

for any $n$. In particular, setting again $\bar{u}_{n}(x):=u_{n}\left(x+\xi_{n}\right)$, for all $\varepsilon>0$ there is $R>0$ such that

$$
\sup _{n \in \mathbb{N}} \int_{B_{R}^{c}(0)}\left|\bar{u}_{n}(x)\right|^{2} d x<\varepsilon .
$$

Assume by contradiction that the sequence $\left\{\xi_{n}\right\}_{n \in \mathbb{N}}$ is unbounded. Then, since $A$ satisfies condition $\mathscr{A}$, there exists a sequence $\left\{H_{n}\right\}_{n \in \mathbb{N}} \subset \mathbb{R}^{3}$ such that (1.4) holds. We thus consider the sequence

$$
v_{n}(x):=e^{\mathrm{i} H_{n} \cdot x} \bar{u}_{n}(x), \quad x \in \mathbb{R}^{3} .
$$

By virtue of Lemma 3.10 it follows that

$$
\sup _{n \in \mathbb{N}}\left\|v_{n}\right\|_{s, A_{n}}=\sup _{n \in \mathbb{N}}\left\|u_{n}\right\|_{s, A}<\infty, \quad A_{n}(x)=A\left(x+\xi_{n}\right)+H_{n} .
$$

Then, by combining Lemma 3.4 with

$$
\sup _{n \in \mathbb{N}} \int_{B_{R}^{c}(0)}\left|v_{n}(x)\right|^{2} d x<\varepsilon
$$

up to a subsequence, $\left\{v_{n}\right\}_{n \in \mathbb{N}}$ is strongly convergent in $L^{q}\left(\mathbb{R}^{3}\right)$ for all $q \in[2,6 /(3-2 s))$ to some function $v$ which satisfies the constraint $\|v\|_{L^{p}}=1$. By combining Lemma 3.10 with Fatou's 
Lemma and (4.7), we get

$$
\begin{aligned}
\mathscr{M}_{A_{\Xi}} \leq\|v\|_{s, A_{\Xi}}^{2} & =\int_{\mathbb{R}^{3}}|v|^{2} d x+\int_{\mathbb{R}^{6}} \frac{\left|e^{-\mathrm{i}(x-y) \cdot A_{\Xi}\left(\frac{x+y}{2}\right)} v(x)-v(y)\right|^{2}}{|x-y|^{3+2 s}} d x d y \\
& \leq \lim _{n} \int_{\mathbb{R}^{3}}\left|v_{n}\right|^{2} d x+\liminf _{n} \int_{\mathbb{R}^{6}} \frac{\left|e^{-\mathrm{i}(x-y) \cdot A_{n}\left(\frac{x+y}{2}\right)} v_{n}(x)-v_{n}(y)\right|^{2}}{|x-y|^{3+2 s}} d x d y \\
& =\lim _{n} \int_{\mathbb{R}^{3}}\left|u_{n}\right|^{2} d x+\liminf _{n} \int_{\mathbb{R}^{6}} \frac{\left|e^{-\mathrm{i}(x-y) \cdot A\left(\frac{x+y}{2}\right)} u_{n}(x)-u_{n}(y)\right|^{2}}{|x-y|^{3+2 s}} d x d y \\
& =\mathscr{M}_{A}<\inf _{\Xi \in \mathscr{X}} \mathscr{M}_{A_{\Xi} \leq \mathscr{M}_{A_{\Xi}}}
\end{aligned}
$$

a contradiction. Therefore, it follows that $\left\{\xi_{n}\right\}_{n \in \mathbb{N}}$ is bounded. The assertion then immediately follows arguing on the original sequence $\left\{u_{n}\right\}_{n \in \mathbb{N}}$.

4.3. Critical case. Let $D_{A}^{s}\left(\mathbb{R}^{3}, \mathbb{C}\right)$ be the completion of $C_{c}^{\infty}\left(\mathbb{R}^{3}, \mathbb{C}\right)$ with respect to the seminorm $[\cdot]_{s, A}$. The functions of $D_{A}^{s}\left(\mathbb{R}^{3}, \mathbb{C}\right)$ satisfy the Sobolev inequality stated in formula (3.1). The space $D_{A}^{s}\left(\mathbb{R}^{3}, \mathbb{C}\right)$ is a real Hilbert space with respect to the scalar product

$$
(u, v)_{s, A}:=\frac{c_{s}}{2} \Re \int_{\mathbb{R}^{6}} \frac{\left(e^{-\mathrm{i}(x-y) \cdot A\left(\frac{x+y}{2}\right)} u(x)-u(y)\right) \overline{\left(e^{-\mathrm{i}(x-y) \cdot A\left(\frac{x+y}{2}\right)} v(x)-v(y)\right)}}{|x-y|^{3+2 s}} d x d y .
$$

We consider the minimization problem $\left(\mathscr{M}_{A}^{c}\right)$. Of course, by density, we have

$$
\mathscr{M}_{A}^{c}=\inf _{u \in \mathscr{S}^{c} \cap C_{c}^{\infty}\left(\mathbb{R}^{3}, \mathbb{C}\right)}[u]_{s, A}^{2}, \quad \mathscr{M}_{0}^{c}=\inf _{u \in \mathscr{S}_{0}^{c} \cap C_{c}^{\infty}\left(\mathbb{R}^{3}, \mathbb{C}\right)}[u]_{s, 0}^{2} .
$$

where $\mathscr{S}_{0}^{c}=\left\{u \in D^{s}\left(\mathbb{R}^{3}, \mathbb{C}\right):\|u\|_{L^{6 /(3-2 s)}}=1\right\}$. Moreover, since $[|u|]_{s, 0} \leq[u]_{s, 0}$, we have

$$
\mathscr{M}_{0}^{c}=\inf _{u \in \mathscr{S}_{0}^{c} \cap C_{c}^{\infty}\left(\mathbb{R}^{3}, \mathbb{R}\right)}[u]_{s, 0}^{2} .
$$

Remark 4.5. It is known $[7,10]$ that all the real valued fixed sign solutions to $\mathscr{M}_{0}^{c}$ are given by

$$
\mathscr{U}_{z, \varepsilon}(x)=d_{s}\left(\frac{\varepsilon}{\varepsilon^{2}+|x-z|^{2}}\right)^{\frac{3-2 s}{2}}
$$

for arbitrary $\varepsilon>0, z \in \mathbb{R}^{3}$ and that these are also the unique fixed sign solutions to

$$
(-\Delta)^{s} u=u^{\frac{3+2 s}{3-2 s}} \text { in } \mathbb{R}^{3} .
$$

We now prove the following crucial lemma

Lemma 4.6. It holds $\mathscr{M}_{A}^{c}=\mathscr{M}_{0}^{c}$.

Proof. Let $\varepsilon>0$ and $u \in C_{c}^{\infty}\left(\mathbb{R}^{3}, \mathbb{R}\right)$ be such that

$$
\int_{\mathbb{R}^{3}}|u|^{6 /(3-2 s)} d x=1, \quad[u]_{s, 0}^{2} \leq \mathscr{M}_{0}^{c}+\varepsilon
$$

in light of formula (4.8) for $\mathscr{M}_{0}^{c}$. Consider now the scaling

$$
u_{\sigma}(x)=\sigma^{-\frac{3-2 s}{2}} u\left(\frac{x}{\sigma}\right), \quad \sigma>0, x \in \mathbb{R}^{3} .
$$

It is readily checked that

$$
\int_{\mathbb{R}^{3}}\left|u_{\sigma}\right|^{6 /(3-2 s)} d x=\int_{\mathbb{R}^{3}}|u|^{6 /(3-2 s)} d x=1, \quad\left[u_{\sigma}\right]_{s, 0}=[u]_{s, 0}, \quad \text { for all } \sigma>0 .
$$


There holds that

$$
\left[u_{\sigma}\right]_{s, A}^{2}=\int_{\mathbb{R}^{6}} \frac{\left|e^{-\mathrm{i} \sigma(x-y) \cdot A\left(\sigma \frac{x+y}{2}\right)} u(x)-u(y)\right|^{2}}{|x-y|^{3+2 s}} d x d y .
$$

Then, we compute

$$
\begin{aligned}
{\left[u_{\sigma}\right]_{s, A}^{2}-[u]_{s, 0}^{2} } & =\int_{\mathbb{R}^{6}} \frac{\left|e^{-\mathrm{i} \sigma(x-y) \cdot A\left(\sigma \frac{x+y}{2}\right)} u(x)-u(y)\right|^{2}-|u(x)-u(y)|^{2}}{|x-y|^{3+2 s}} d x d y \\
& =\int_{\mathbb{R}^{6}} \Theta_{\sigma}(x, y) d x d y=\int_{K \times K} \Theta_{\sigma}(x, y) d x d y
\end{aligned}
$$

where $K$ is the compact support of $u$ and

$$
\begin{aligned}
\Theta_{\sigma}(x, y) & :=\frac{2 \Re\left(\left(1-e^{-\mathrm{i} \sigma(x-y) \cdot A\left(\sigma \frac{x+y}{2}\right)}\right) u(x) u(y)\right)}{|x-y|^{3+2 s}} \\
& =\frac{2\left(1-\cos \left(\sigma(x-y) \cdot A\left(\sigma \frac{x+y}{2}\right)\right)\right) u(x) u(y)}{|x-y|^{3+2 s}},
\end{aligned}
$$

a.e. in $\mathbb{R}^{6}$. Of course $\Theta_{\sigma}(x, y) \rightarrow 0$ for a.e. $(x, y) \in \mathbb{R}^{6}$ as $\sigma \rightarrow 0$. Since $A$ is locally bounded then

$$
1-\cos \left(\sigma(x-y) \cdot A\left(\sigma \frac{x+y}{2}\right)\right) \leq C|x-y|^{2} \quad x, y \in K
$$

Therefore, since $u$ is bounded, it follows that for some $C>0$

$$
\begin{aligned}
& \left|\Theta_{\sigma}(x, y)\right| \leq \frac{C}{|x-y|^{1+2 s}}, \quad \text { for } x, y \in K \text { with }|x-y|<1, \\
& \left|\Theta_{\sigma}(x, y)\right| \leq \frac{C}{|x-y|^{3+2 s}}, \quad \text { for } x, y \in K \text { with }|x-y| \geq 1 .
\end{aligned}
$$

Then overall, we have

$$
\left|\Theta_{\sigma}(x, y)\right| \leq w(x, y), \quad w(x, y)=C \min \left\{\frac{1}{|x-y|^{1+2 s}}, \frac{1}{|x-y|^{3+2 s}}\right\} \quad \text { for } x, y \in K,
$$

for a suitable constant $C>0$. Notice that $w \in L^{1}(K \times K)$, since

$$
\begin{aligned}
\int_{K \times K} w(x, y) d x d y & =\int_{(K \times K) \cap\{|x-y|<1\}} w(x, y) d x d y+\int_{(K \times K) \cap\{|x-y| \geq 1\}} w(x, y) d x d y \\
& \leq C \int_{\{|z|<1\}} \frac{1}{|z|^{1+2 s}} d z+C \int_{\{|z| \geq 1\}} \frac{1}{|z|^{3+2 s}} d z<\infty .
\end{aligned}
$$

Then, by the Dominated Convergence Theorem, we obtain

$$
\mathscr{M}_{A}^{c} \leq \lim _{\sigma \rightarrow 0}\left[u_{\sigma}\right]_{s, A}^{2}=[u]_{s, 0}^{2} \leq \mathscr{M}_{0}^{c}+\varepsilon,
$$

hence $\mathscr{M}_{A}^{c} \leq \mathscr{M}_{0}^{c}$ by the arbitrariness of $\varepsilon$. Since the opposite inequality is trivial through the Diamagnetic inequality, the desired assertion follows.

Thus we can prove (i) of Theorem 1.3.

Theorem 4.7 (Representation of solutions). Assume that $\mathscr{M}_{A}^{c}$ admits a solutions $u \in D_{A}^{s}\left(\mathbb{R}^{3}, \mathbb{C}\right)$. Then there exist $z \in \mathbb{R}^{3}, \varepsilon>0$ and a function $\vartheta_{A}: \mathbb{R}^{3} \rightarrow \mathbb{R}$ such that

$$
u(x)=d_{s}\left(\frac{\varepsilon}{\varepsilon^{2}+|x-z|^{2}}\right)^{\frac{3-2 s}{2}} e^{\mathrm{i} \vartheta_{A}(x)}, \quad x \in \mathbb{R}^{3} .
$$


Proof. If $u \in D_{A}^{s}\left(\mathbb{R}^{3}, \mathbb{C}\right)$ is a solution to $\mathscr{M}_{A}^{c}$, then by the Diamagnetic inequality and Lemma 4.6,

$$
\mathscr{M}_{A}^{c}=\mathscr{M}_{0}^{c} \leq[|u|]_{s, 0}^{2} \leq[u]_{s, A}^{2}=\mathscr{M}_{A}^{c} .
$$

Then, it follows that $\mathscr{M}_{0}^{c}=[|u|]_{s, 0}^{2}$, which implies the assertion by Remark 4.5.

For a function $u \in D_{A}^{s}\left(\mathbb{R}^{3}, \mathbb{C}\right)$ we define $\Upsilon_{u}^{A}: \mathbb{R}^{6} \rightarrow \mathbb{R}$ by setting

$$
\Upsilon_{u}^{A}(x, y):=2 \Re\left(|u(x)||u(y)|-e^{-\mathrm{i}(x-y) \cdot A\left(\frac{x+y}{2}\right)} u(x) \bar{u}(y)\right), \quad \text { a.e. in } \mathbb{R}^{6} .
$$

Finally we have

Theorem 4.8 (Nonexistence). Assume that for a function $u \in D_{A}^{s}\left(\mathbb{R}^{3}, \mathbb{C}\right)$ we have

$$
\Upsilon_{u}^{A}(x, y)>0 \text { on } E \subset \mathbb{R}^{6} \text { with } \mathcal{L}^{6}(E)>0 \text {. }
$$

Then $u$ cannot be a solution to problem $\mathscr{M}_{A}^{c}$.

Proof. For every $u \in D_{A}^{s}\left(\mathbb{R}^{3}, \mathbb{C}\right)$ we have $|u| \in D^{s}\left(\mathbb{R}^{3}\right)$ and there holds

$$
[u]_{s, A}^{2}-[|u|]_{s, 0}^{2}=\int_{\mathbb{R}^{6}} \Upsilon_{u}^{A}(x, y) d x d y
$$

Assume by contradiction that $u$ solves $\mathscr{M}_{A}^{c}$. Then, since $\|u\|_{L^{6 /(3-2 s)}}=1$, by Lemma 4.6 and assumption (4.9), we conclude that $\mathscr{M}_{0}^{c}=\mathscr{M}_{A}^{c}=[u]_{s, A}^{2}>[|u|]_{s, 0}^{2} \geq \mathscr{M}_{0}^{c}$, a contradiction.

As a consequence we get (ii) of Theorem 1.3.

Corollary 4.9 (Nonexistence of constant phase solutions). Assume that

$$
(x-y) \cdot A(x+y) \not \equiv k \pi, \quad \text { for some } k \in \mathbb{N} \text { and on some } E \subset \mathbb{R}^{6} \text { with } \mathcal{L}^{6}(E)>0 .
$$

Then $\mathscr{M}_{A}^{c}$ does not admit solutions $u \in D_{A}^{s}\left(\mathbb{R}^{3}, \mathbb{C}\right)$ of the form $u(x)=e^{\mathrm{i} \vartheta} v(x)$ for some $\vartheta \in \mathbb{R}$ and $v \in D_{A}^{s}\left(\mathbb{R}^{3}, \mathbb{R}\right)$ of fixed sign.

Proof. Assumption (4.9) is fulfilled, since

$$
\Upsilon_{u}^{A}(x, y)=2\left(1-\cos \left((x-y) \cdot A\left(\frac{x+y}{2}\right)\right)\right) v(x) v(y)>0, \quad \text { for a.e. }(x, y) \in E .
$$

Hence, the assertion follows from Theorem 4.8.

\section{REFERENCES}

[1] A. Applebaum, Lévy processes and Stochastic Calculus, Cambridge Studies in Advanced Mathematics 116, Cambridge University Press, Cambridge, 2009. 2

[2] G. Arioli, A. Szulkin, A semilinear Schrödinger equation in the presence of a magnetic field, Arch. Ration. Mech. Anal. 170 (2003), 277-295. 2

[3] J. Avron, I. Herbst, B. Simon, Schrödinger operators with magnetic fields. I. General interactions, Duke Math. J. 45 (1978), 847-883. 2

[4] L. Brasco, E. Parini, The second eigenvalue of the fractional p-Laplacian, Adv. Calc. Var. 9 (2016), 323-355. 10

[5] J. Bourgain, H. Brezis and P. Mironescu, Another look at Sobolev spaces, in Optimal Control and Partial Differential Equations. A Volume in Honor of Professor Alain Bensoussan's 60th Birthday (eds. J. L. Menaldi, E. Rofman and A. Sulem), IOS Press, Amsterdam, 2001, 439-455. 2, 8

[6] J. Bourgain, H. Brezis and P. Mironescu, Limiting embedding theorems for $W^{s, p}$ when $s \uparrow 1$ and applications, J. Anal. Math. 87 (2002), 77-101. 2, 8

[7] W. Chen, C. Li, B. Ou, Classification of solutions for an integral equation, Comm. Pure Appl. Math. 59 (2006), 330-343. 19

[8] S. Cingolani, S. Secchi, Semiclassical limit for nonlinear Schrödinger equations with electromagnetic fields, J. Math. Anal. Appl. 275 (2002), 108-130. 2 
[9] R. Cont, P. Tankov, Financial modeling with jump processes, Chapman \& Hall/CRC Financial Mathematics Series, Chapman \& Hall/CRC, Boca Raton, FL, 2004. 2

[10] A. Cotsiolis, N.K. Tavoularis, Best constants for Sobolev inequalities for higher order fractional derivatives, J. Math. Anal. Appl. 295 (2004), 225-236. 19

[11] P. d'Avenia, G. Siciliano, M. Squassina, On fractional Choquard equations, Math. Models Methods Appl. Sci. 25 (2015), 1447-1476. 10

[12] J. Di Cosmo, J. Van Schaftingen, Semiclassical stationary states for nonlinear Schrödinger equations under a strong external magnetic field, J. Differential Equations 259 (2015), 596-627. 2

[13] E. Di Nezza, G. Palatucci, E. Valdinoci, Hitchhiker's guide to the fractional Sobolev spaces, Bull. Sci. Math. 136 (2012), 521-573. 9, 10

[14] S. Dipierro, G. Palatucci, E. Valdinoci, Existence and symmetry results for a Schrödinger type problem involving the fractional Laplacian, Le Matematiche 68 (2013), 201-216. 4

[15] M. Esteban, P.L. Lions, Stationary solutions of nonlinear Schrödinger equations with an external magnetic field, Partial differential equations and the calculus of variations, Vol. I, 401-449, Progr. Nonlinear Differential Equations Appl. 1, Birkhäuser Boston, Boston, MA, 1989. 2, 4, 16

[16] R.L. Frank, E.H. Lieb, R. Seiringer, Hardy-Lieb-Thirring inequalities for fractional Schrödinger operators, J. Amer. Math. Soc. 21 (2008), 925-950. 2

[17] T. Ichinose, Essential selfadjointness of the Weyl quantized relativistic Hamiltonian, Ann. Inst. H. Poincaré Phys. Théor. 51 (1989), 265-297. 2

[18] T. Ichinose, Magnetic relativistic Schrödinger operators and imaginary-time path integrals, Mathematical physics, spectral theory and stochastic analysis, 247-297, Oper. Theory Adv. Appl. 232, Birkhäuser/Springer Basel AG, Basel, 2013. 2, 3

[19] T. Ichinose, H. Tamura, Imaginary-time path integral for a relativistic spinless particle in an electromagnetic field, Comm. Math. Phys. 105 (1986), 239-257. 2

[20] V. Iftimie, M. Măntoiu, R. Purice, Magnetic pseudodifferential operators, Publ. Res. Inst. Math. Sci. 43 (2007), 585-623. 2

[21] K. Kurata, Existence and semi-classical limit of the least energy solution to a nonlinear Schrödinger equation with electromagnetic fields, Nonlinear Anal. 41 (2000), 763-778. 2

[22] N. Laskin, Fractional Schrödinger equation, Phys. Rev. E 66 (2002), 056108. 2

[23] E.H. Lieb, R. Seiringer, The stability of matter in quantum mechanics, Cambridge University Press, Cambridge, 2010. 2

[24] P.-L. Lions, Symétrie and compacité dans le espaces de Sobolev, J. Funct. Anal 49 (1982), 315-334. 15

[25] P.-L. Lions, The concentration-compactness principle in the calculus of variation. The locally compact case I, Ann. Inst. H. Poincaré Anal. Non Linéaire 1 (1984), 109-145. 16, 17

[26] R. Metzler, J. Klafter, The restaurant at the random walk: recent developments in the description of anomalous transport by fractional dynamics, J. Phys. A 37 (2004), R161-R208. 2

[27] M. Reed, B. Simon, Methods of modern mathematical physics, I, Functional analysis, Academic Press, Inc., New York, 1980. 2

[28] M. Squassina, Soliton dynamics for the nonlinear Schrödinger equation with magnetic field, Manuscripta Math. 130 (2009), 461-494. 2

(P. d'Avenia) Dipartimento di Meccanica, Matematica e Management

Politecnico di Bari, Via Orabona 4, I-70125 Bari, Italy

E-mail address: pietro.davenia@poliba.it

(M. Squassina) Dipartimento di Matematica e Fisica

Università Cattolica del Sacro Cuore, Via dei Musei 41, I-25121 Brescia, Italy

E-mail address: marco.squassina@unicatt.it 